\title{
Multi-wavelength Observations of AT2019wey: a New Candidate Black Hole Low-mass X-ray Binary
}

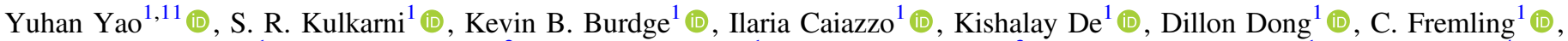
Mansi M. Kasliwal ${ }^{1}$ (i), Thomas Kupfer ${ }^{2}$ (i), Jan van Roestel ${ }^{1}$ (10), Jesper Sollerman ${ }^{3}$ (1), Ashot Bagdasaryan ${ }^{1}$, Eric C. Bellm ${ }^{4}$ (1), S. Bradley Cenko ${ }^{5}$ (i), Andrew J. Drake ${ }^{1}$, Dmitry A. Duev ${ }^{1}\left(\mathbb{1}\right.$, Matthew J. Graham ${ }^{1}$ (i), Stephen Kaye ${ }^{6}$, Frank J. Masci ${ }^{7}$,

Nicolas Miranda ${ }^{8}$ (i), Thomas A. Prince ${ }^{1}$ (1), Reed Riddle ${ }^{6}$ (i), Ben Rusholme ${ }^{7}$ (i), and Maayane T. Soumagnac ${ }^{9,10}$ (1)

${ }^{1}$ Division of Physics, Mathematics and Astronomy, California Institute of Technology, Pasadena, CA 91125, USA; yyao@astro.caltech.edu

${ }^{2}$ Texas Tech University, Department of Physics \& Astronomy, Box 41051, 79409, Lubbock, TX, USA

${ }^{3}$ The Oskar Klein Centre, Department of Astronomy, Stockholm University, AlbaNova, SE-10691 Stockholm, Sweden

${ }^{4}$ DIRAC Institute, Department of Astronomy, University of Washington, 3910 15th Avenue NE, Seattle, WA 98195, USA

${ }^{5}$ Astrophysics Science Division, NASA Goddard Space Flight Center, Greenbelt, MD 20771, USA

${ }^{6}$ Caltech Optical Observatories, California Institute of Technology, Pasadena, CA 91125, USA

${ }^{7}$ IPAC, California Institute of Technology, 1200 E. California Blvd, Pasadena, CA 91125, USA

${ }^{8}$ Institut für Informatik, Humboldt-Universität zu Berlin, Rudower Chaussee 25, D-12489 Berlin, Germany

${ }^{9}$ Lawrence Berkeley National Laboratory, 1 Cyclotron Road, Berkeley, CA 94720, USA

${ }^{10}$ Department of Particle Physics and Astrophysics, Weizmann Institute of Science, Rehovot 76100, Israel

Received 2020 November 30; revised 2021 July 2; accepted 2021 July 16; published 2021 October 21

\begin{abstract}
AT2019wey (SRGA J043520.9+552226, SRGE J043523.3+552234) is a transient first reported by the ATLAS optical survey in 2019 December. It rose to prominence upon detection, three months later, by the SpektrumRoentgen-Gamma (SRG) mission in its first all-sky survey. X-ray observations reported in Yao et al. suggest that AT2019wey is a Galactic low-mass X-ray binary (LMXB) with a black hole (BH) or neutron star (NS) accretor. Here we present ultraviolet, optical, near-infrared, and radio observations of this object. We show that the companion is a short-period $(P \lesssim 16 \mathrm{hr})$ low-mass $\left(<1 M_{\odot}\right)$ star. We consider AT2019wey to be a candidate BH system since its locations on the $L_{\mathrm{radio}}-L_{\mathrm{X}}$ and $L_{\mathrm{opt}}-L_{\mathrm{X}}$ diagrams are closer to BH binaries than NS binaries. We demonstrate that from 2020 June to August, despite the more than 10 times brightening at radio and X-ray wavelengths, the optical luminosity of AT2019wey only increased by 1.3-1.4 times. We interpret the UV/optical emission before the brightening as thermal emission from a truncated disk in a hot accretion flow and the UV/ optical emission after the brightening as reprocessing of the X-ray emission in the outer accretion disk. AT2019wey demonstrates that combining current wide-field optical surveys and SRG provides a way to discover the emerging population of short-period BH LMXB systems with faint X-ray outbursts.
\end{abstract}

Unified Astronomy Thesaurus concepts: Low-mass x-ray binary stars (939); Accretion (14); Astrophysical black holes (98); Sky surveys (1464)

Supporting material: machine-readable table

\section{Introduction}

\subsection{Low-mass X-ray Binaries and SRG}

Low-mass X-ray binaries (LMXBs) contain an accreting neutron star (NS) or black hole (BH) in orbit with a low-mass $\left(\lesssim 2 M_{\odot}\right)$ companion star. Most of the known BH LMXBs were discovered by X-ray all-sky monitors (ASMs) during X-ray outbursts induced by instabilities in the accretion processes. The most sensitive X-ray ASM to date, the Monitor of All-sky X-ray Image (MAXI; Matsuoka et al. 2009), has a transient triggering threshold of $8 \mathrm{mCrab}\left(1 \mathrm{mCrab}=2.4 \times 10^{-11} \mathrm{erg} \mathrm{s}^{-1} \mathrm{~cm}^{-2}\right.$ over 2-10 keV) sustained for 4 days (Negoro et al. 2016). Due to the relatively shallow sensitivity of ASMs, the sample of LMXBs is biased toward nearby sources that exhibit bright $\mathrm{X}$-ray outbursts.

Prior to 2020, the most sensitive all-sky X-ray imaging survey was carried out in 1990/1991 by ROSAT at $0.1-2.4 \mathrm{keV}$ (Truemper 1982; Voges et al. 1999). It cataloged X-ray sources brighter than $\sim 10 \mu \mathrm{Crab}$, providing the deepest X-ray all-sky reference at the time (Boller et al. 2016). Three decades after ROSAT, the dynamic X-ray sky is being surveyed by the

\footnotetext{
${ }^{11}$ Author to whom any correspondence should be addressed.
}

eROSITA (0.2-10 keV; Predehl et al. 2021) and the Mikhail Pavlinsky ART-XC (4-30 keV; Pavlinsky et al. 2021) telescopes on board the Spektrum-Roentgen-Gamma (SRG) mission (Sunyaev et al. 2021). This planned four-year survey obtaining full-sky images created every six months is a powerful X-ray time-domain facility. The first eROSITA All-Sky Survey (eRASS1; 2019 December-2020 June) was sensitive to point sources down to $\sim 0.8 \mu \mathrm{Crab}$ (Predehl et al. 2021).

\subsection{AT2019wey}

On 2020 March 18, SRG discovered a new X-ray ( $1 \mathrm{mCrab})$ transient，SRGA J043520.9+552226 (=SRGE J043523.3+ 552234; Mereminskiy et al. 2020). It coincided with an optical $(r \sim 17.5)$ transient, AT2019wey, first reported by ATLAS (Tonry et al. 2019). This transient, bright at both X-ray and optical wavelengths and located at low Galactic latitude, $\left(b=5^{\circ} .3\right)$ was not present in the Palomar Observatory Sky Survey or the ROSAT catalog. We conducted an extensive follow-up campaign, revealing that AT2019wey is a Galactic LMXB with unique properties.

Yao et al. (2020a, hereafter Paper I) presented X-ray observations of AT2019wey from 2019 January to 2020 


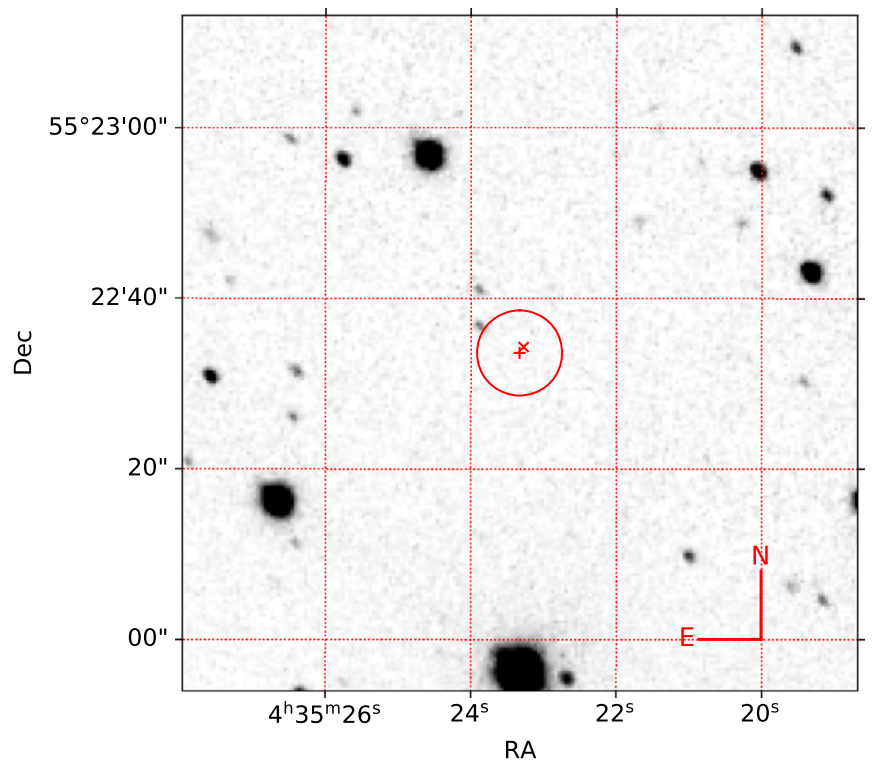

Figure 1. Localization of AT2019wey plotted on top of the SDSS $z$-band image. The eROSITA and ZTF position is shown by "+" sign and " $\times$ ", respectively. The circle indicates eROSITA's $68 \%$ error radius of $5^{\prime \prime}$ (Mereminskiy et al. 2020). The ZTF position is R.A $=04^{\mathrm{h}} 35^{\mathrm{m}} 23^{\mathrm{s}} .27$, decl. $=+55^{\mathrm{d}} 22^{\mathrm{m}} 34^{\mathrm{s}} .3(\mathrm{~J} 2000)$.

November, suggesting that AT2019wey is an LMXB with a $\mathrm{BH}$ or NS accretor. In this work, we present multi-wavelength observations of AT2019wey. We conclude that the compact object is probably a BH and the companion star must be of low mass $\left(<1 M_{\odot}\right)$. We therefore call AT2019wey a candidate $\mathrm{BH}$ LMXB. This class of objects and the classification of their X-ray states is reviewed in McClintock \& Remillard (2006), Remillard \& McClintock (2006), Belloni et al. (2011), Zhang (2013), and Tetarenko et al. (2016).

The paper is organized as follows. The association between the optical and X-ray transients is outlined in Section 2. We present optical and ultraviolet (UV) photometry in Section 3, optical and near-infrared (NIR) spectroscopy in Section 4, and radio observations in Section 5. We discuss the nature of the source in Section 6, and summarize our findings and conclusions in Section 7.

Throughout this paper, times are reported in UT. Optical magnitudes are reported in the $\mathrm{AB}$ system. We adopt the reddening law of Cardelli et al. (1989) with $R_{V}=3.1$.

\section{Association between the Optical and X-ray Transients}

On 2019 December 2 05:18:40 (MJD 58819.2213), the Zwicky Transient Facility (ZTF; Bellm et al. 2019; Graham et al. 2019) detected AT2019wey at a $g$-band $\left(\lambda_{\text {eff }}=4810 \AA\right)$ magnitude of $g_{\text {ZTF }}=19.30 \pm 0.05$. The last non-detection was obtained by ATLAS at an $o$-band $\left(\lambda_{\text {eff }}=6790 \AA\right)$ magnitude of $o_{\text {ATLAS }}>$ 18.3, on 1 December 2019 12:18:30 (MJD 58818.5129).

In Figure 1 we display the X-ray localization on an optical image. The AT2019wey and SRGE J043523.3+552234 locations are separated by only $0 . / 8$, well within the X-ray error radius, thereby confirming the association first suggested by Mereminskiy et al. (2020). The Galactic coordinates of AT2019wey, $l=151^{\circ} .2$ and $b=5^{\circ} .3$, a priori favors a Galactic source in the Galactic anti-center direction.

\section{Photometry}

\subsection{ZTF, ATLAS, and Gaia Photometry}

We constructed the optical light curve using the forcedphotometry services of $\mathrm{ZTF}^{12}$ (Masci et al. 2019) and $\operatorname{ATLAS}^{13}$ (Smith et al. 2020). We obtained Gaia photometry from the Gaia alerts page. ${ }^{14}$ See Table 5 for the ZTF photometry.

The upper panel of Figure 2 shows the ZTF, ATLAS, and Gaia light curves of AT2019wey. Over the first two weeks, the light curve rose to $r_{\mathrm{ZTF}}=17.3 \mathrm{mag}$. After that, the light curve displayed small amplitude ( $\lesssim 0.3 \mathrm{mag}$ ) variability for more than 300 days. The lack of photometry between MJD $~ 58980$ to MJD 59040 is due to the source being in the day sky. On September 9 and 13 we undertook continuous observations as part of the ZTF “deep-drilling” program (Kupfer et al. 2021). On each day, $\approx 130 r$-band exposure frames were obtained.

\subsection{CHIMERA Photometry}

On 2020 July 23 (MJD 59053), we obtained high-speed photometry in the SDSS $g$ and $i$ band using the Caltech HIghspeed Multi-color camERA (CHIMERA; Harding et al. 2016) on the 200-inch Hale telescope of the Palomar Observatory. We operated the detectors using the $1 \mathrm{MHz}$ conventional amplifier in frame-transfer mode with a frame exposure time of $1 \mathrm{~s}$, and obtained 3300 frames in each filter. We reduced the data with a custom pipeline. ${ }^{15}$ Figure 3 shows the CHIMERA light curve. AT2019wey appears to exhibit intra-night variability of $\sim 0.1 \mathrm{mag}$.

\subsection{Period Search}

We ran a periodicity search on the CHIMERA and the ZTF deep-drilling data sets using the analysis of variance (AOV) method (Schwarzenberg-Czerny 1998). ${ }^{16}$ We used a frequency grid from 16 day $^{-1}$ to 500 day $^{-1}$ for the ZTF data and a frequency grid from 48 day $^{-1}$ to 40,000 day $^{-1}$ for the CHIMERA data. To see how the observational cadence affects the periodogram, we used the Lomb-Scargle algorithm (see a recent review by VanderPlas 2018) to compute the window function.

We define "significance" of a period as the maximum value in the periodogram divided by the standard deviation of values across the full periodogram. A possible period at 0.055 day $(1.3 \mathrm{hr})$ at a significance of 9.2 can be seen in the ZTF periodogram (upper panel of Figure 4). We note that the $1.3 \mathrm{hr}$ peak is mainly caused by the sinusoidal-like structure observed on September 19, not the dip-like structure observed on September 23. Since the data on September 19 and 23 do not follow the same trend as a function of phase (see lower panel of Figure 4), we consider the possible period at $1.3 \mathrm{hr}$ to be spurious. No period above $8 \sigma$ can be identified from the CHIMERA periodogram.

\footnotetext{
12 https://ztfweb.ipac.caltech.edu/cgi-bin/requestForcedPhotometry.cgi

13 https://fallingstar-data.com/forcedphot/

$14 \mathrm{http}: / /$ gsaweb.ast.cam.ac.uk/alerts/alert/Gaia20aua/

15 https://github.com/mcoughlin/kp84

${ }^{16}$ We used the python script provided by https://users.camk.edu.pl/alex/ \#software.
} 


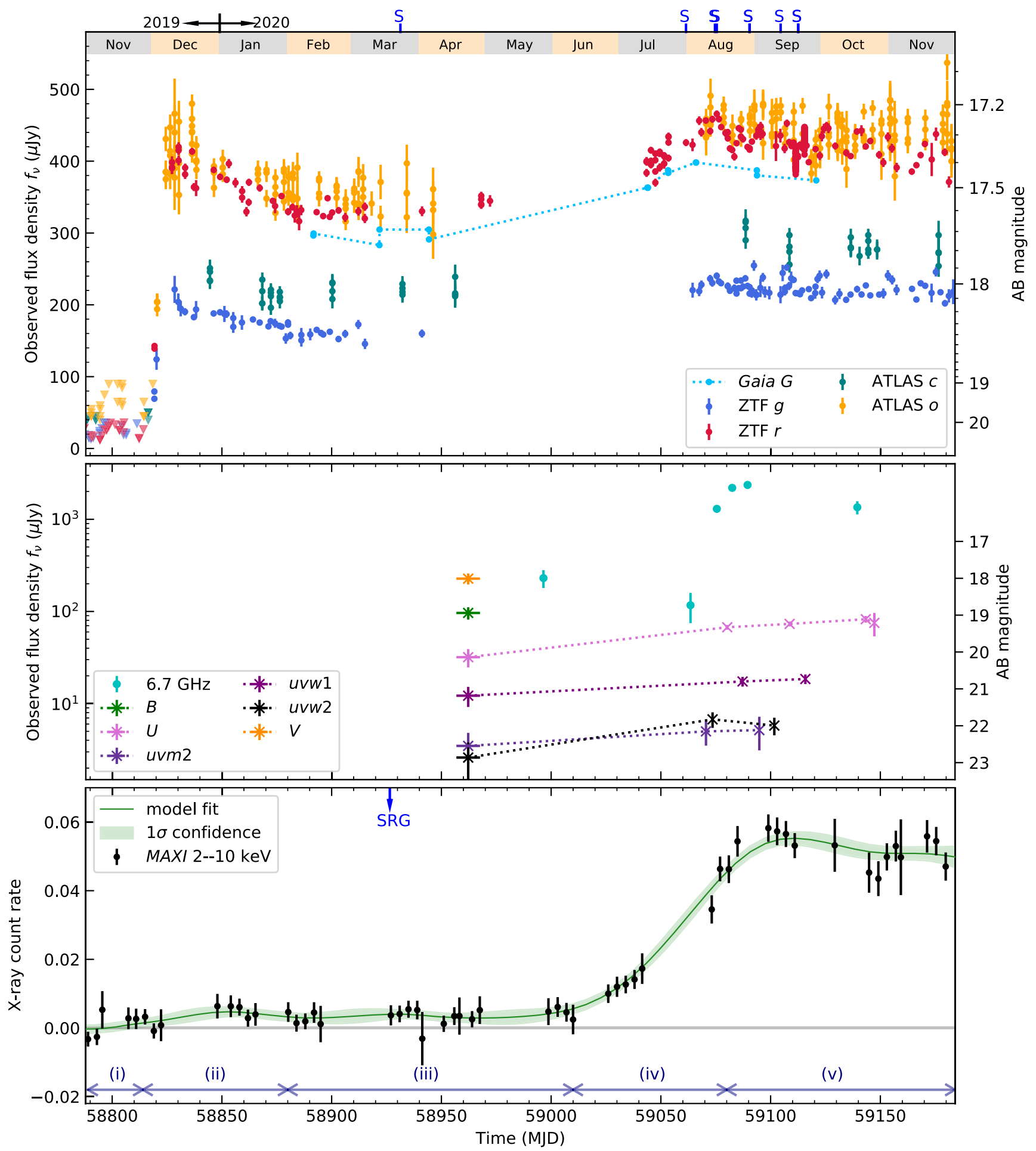

Figure 2. Multi-wavelength light curves of AT2019wey. Upper limits are shown as downward triangles. Upper: optical light curves from ZTF, ATLAS, and Gaia (Section 3.1). Epochs of spectroscopy (Table 1) are marked with the letter S above the upper axis. Middle: UV (Section 3.4) and radio (Section 5) light curves. The $6.7 \mathrm{GHz}$ flux densities are interpolated from the power-law fits in Table 2. Bottom: MAXI 2-10 keV light curve (Paper I). The green curve is a model fit to the data, generated using a Gaussian process following procedures described in Appendix B.4 of Yao et al. (2020b). The epoch of SRG discovery is marked by the blue arrow. The multi-wavelength evolution is divided into five stages (see discussion in Section 6.2).

\subsection{UV Photometry}

We obtained UV observations of AT2019wey with the UltraViolet/Optical Telescope (UVOT; Roming et al. 2005) on board the Neil Gehrels Swift Observatory (Gehrels et al. 2004) from 2020 April to September. The UVOT data were processed using HEASOft. We extracted the photometry with uvotsource using a $3^{\prime \prime}$ circular aperture. Background counts were estimated in a $10^{\prime \prime}$ source-free circular aperture. AT2019wey was only marginally detected in April. Therefore, for the April data sets, we undertook photometry on co-added images.

In October 2020, we obtained $U$-band photometry using the Spectral Energy Distribution Machine (SEDM, 


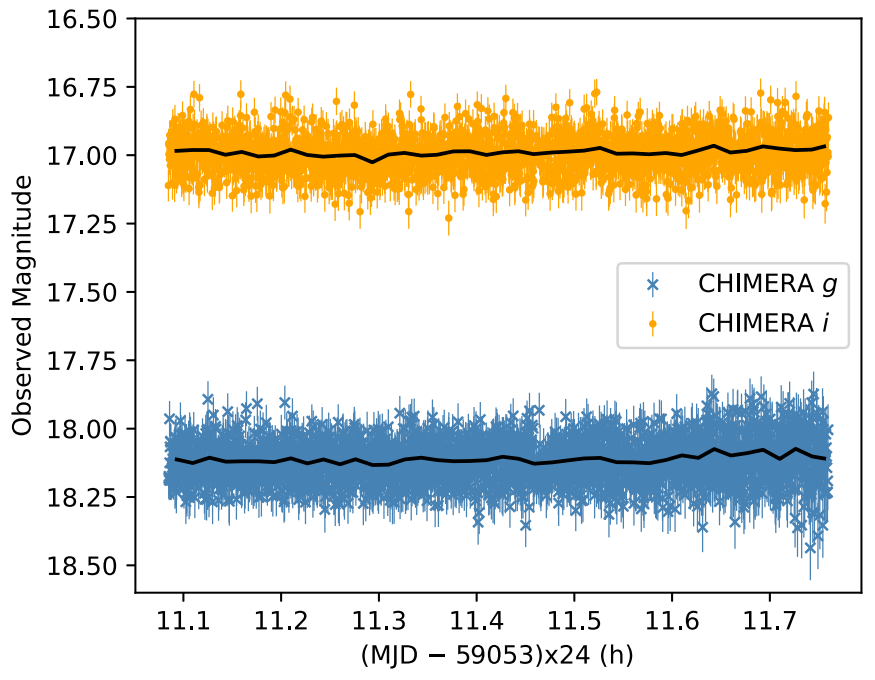

Figure 3. CHIMERA data of AT2019wey. The black lines show light curves averaged to 1 minute. The median magnitudes are $i=16.99 \pm 0.07$ and $g=18.12 \pm 0.08$. The $g$-band rms increased toward the end of the observation due to the onset of twilight.

Blagorodnova et al. 2018; Rigault et al. 2019) on the robotic Palomar 60 inch telescope (P60, Cenko et al. 2006). Data reduction was performed using the FPipe pipeline (Fremling et al. 2016). The UVOT and SEDM photometry are presented in Table 6 and a shown in the middle panel of Figure 2.

\section{Optical and NIR Spectroscopy}

A $\log$ of our spectroscopic observations is given in Table 1. The instrumental and observational details can be found in Appendix B.

\subsection{Optical Spectroscopy}

We identify the following features at redshift $z=0$ in all of our spectra: Balmer absorption lines, $\mathrm{Ca}$ II $\mathrm{H}$ and $\mathrm{K}$ lines, the $\mathrm{Na}$ I D doublet, diffuse interstellar band (DIB) $\lambda 5780, \lambda 6283$ absorption features, and the Balmer jump (Figure 5, 6). He II $\lambda 4686$ emission seems to be detected in the spectra obtained on July 31, August 14, and September 20. We conclude that AT2019wey is a transient of Galactic stellar origin.

From March to September, the hydrogen profile clearly changed (Figure 6). Figure 7 presents the velocity profiles of Balmer lines in the March 23 and the September 12 spectra. On March 23, we observed a relatively narrower $\left(\mathrm{FWHM} \sim 1200 \mathrm{~km} \mathrm{~s}^{-1}\right.$ ) emission component in the middle of a rotationally broadened (FWHM $\sim$ $2700 \mathrm{~km} \mathrm{~s}^{-1}$ ) shallow absorption trough. At the same epoch, we also observed broad $\mathrm{H} \beta$ and $\mathrm{H} \gamma$ absorption features with FWHM $\sim 2000-3000 \mathrm{~km} \mathrm{~s}^{-1}$. There was a marginal detection of narrow emission cores redshifted by $\sim 300-400 \mathrm{~km} \mathrm{~s}^{-1}$ from the line center of the absorption troughs. On September 12, we observed flat-topped $\mathrm{H} \alpha$ in emission $\left(\sim 400 \mathrm{~km} \mathrm{~s}^{-1}\right)$, while the $\mathrm{H} \beta$ and $\mathrm{H} \gamma$ profiles were similar to the $\mathrm{H} \alpha$ profile on March 23. The variable Balmer features are discussed further in Section 6.4.

The reddening of AT2019wey can be constrained to $0.8<$ $E(B-V)<1.2$ (Appendix C.1) using the equivalent width $(\mathrm{EW})$ of the interstellar absorption lines. We find a lower limit to the distance of AT2019wey of $D>1 \mathrm{kpc}$ using the velocities of the NaI doublet in the ESI spectrum (Appendix C.2). In addition, since AT2019wey is in the Galactic anti-center direction, the distance to AT2019wey is likely less than
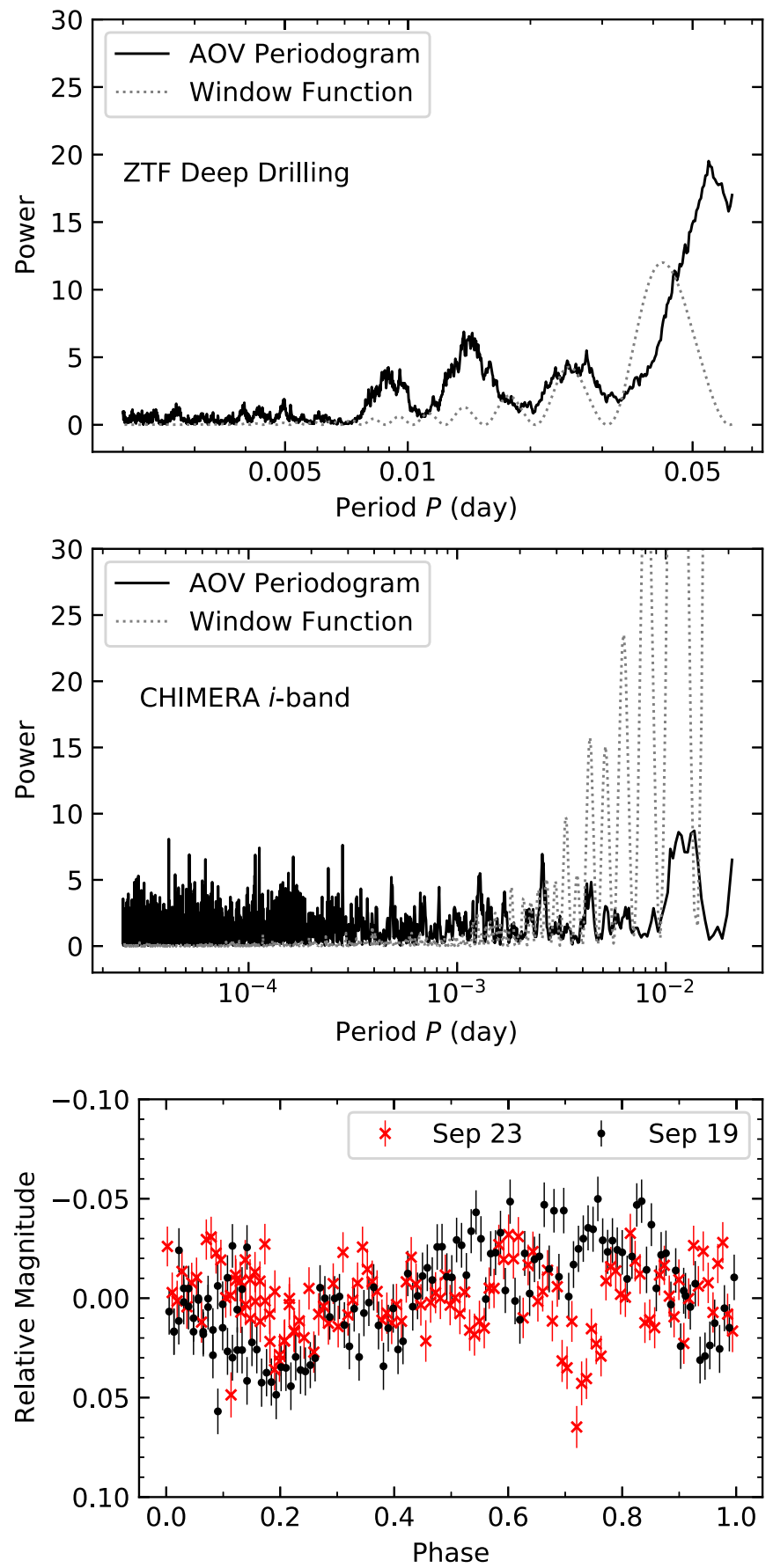

Figure 4. Upper: the periodogram and window function for the ZTF deepdrilling data set. Middle: the periodogram and window function for CHIMERA $i$ band. Note that the periodogram for the $g$-band data is similar to that of the $i$ band. Bottom: the ZTF deep-drilling light curve, relative to the median, folded on a period of $0.055 \mathrm{~d}$.

$\sim 10 \mathrm{kpc}$. Taken together, we conclude that the distance of AT2019wey is between $\sim 1 \mathrm{kpc}$ and $\sim 10 \mathrm{kpc}$.

\subsection{NIR Spectroscopy}

The NIR spectrum of AT2019wey is shown in Figure 8. Hydrogen emission lines of $\operatorname{Pa} \gamma, \operatorname{Pa} \beta$, and $\mathrm{Br} \gamma$ are clearly distinguished. We tentatively attribute the emission lines around $1083 \mathrm{~nm}$ to double-peaked He I. No absorption lines or molecular bands from the secondary star can be identified. With an FWHM of $\approx 200-300 \mathrm{~km} \mathrm{~s}^{-1}$, the velocities of NIR 


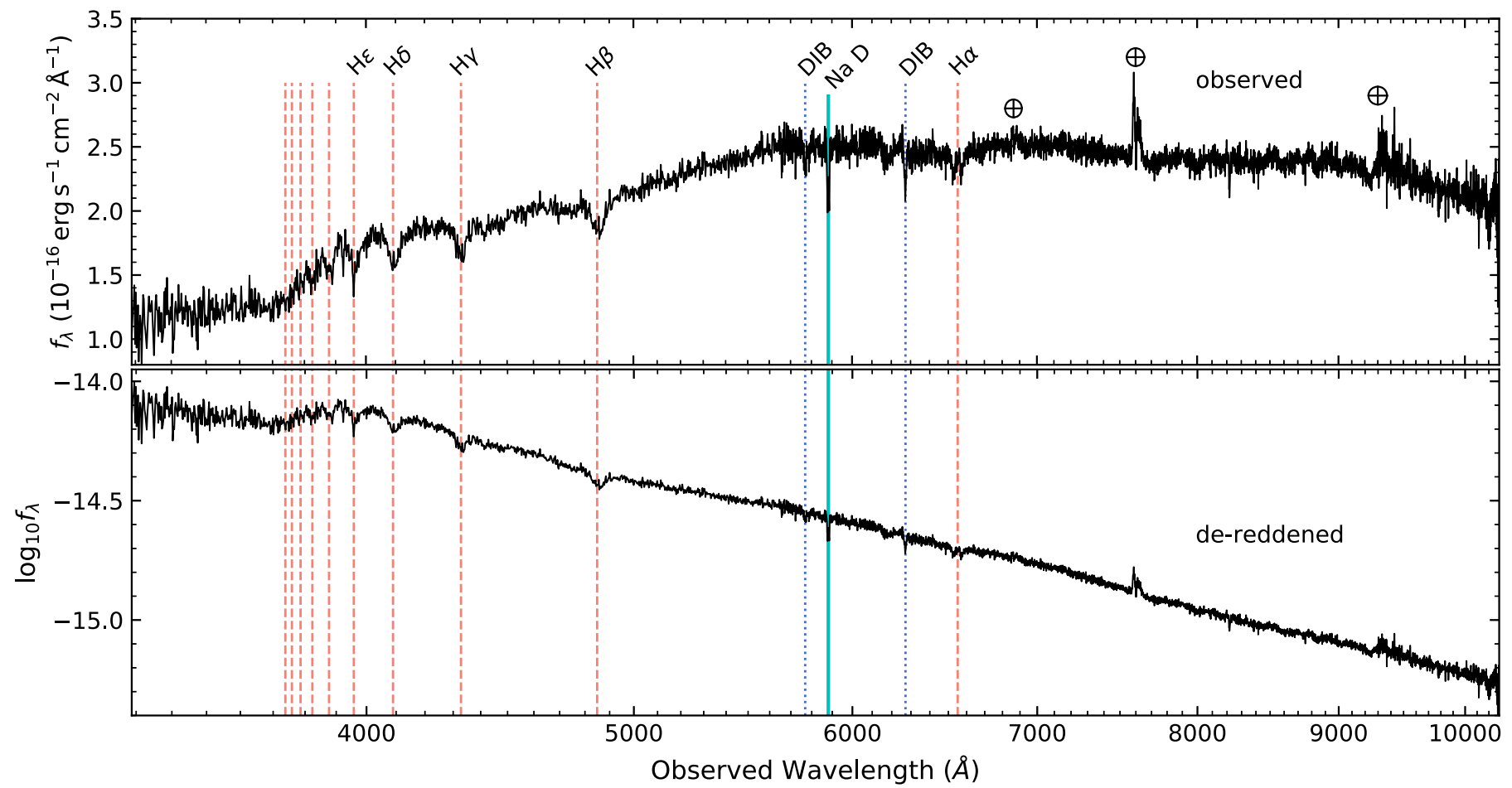

Figure 5. LRIS spectrum of AT2019wey obtained on 2020 March 18. Upper: observed spectrum. Bottom: extinction-corrected spectrum using $E(B-V)=0.9$. Rest (air) wavelength of atomic transitions is marked with vertical lines.

Table 1

Log of AT2019wey Spectroscopy

\begin{tabular}{|c|c|c|c|c|}
\hline Date in 2020 & Telescope/Instrument & Range $(\AA)$ & Exp. (s) & Air Mass \\
\hline Mar 23 & Keck I/LRIS & $3200-10250$ & 300 & 2.22 \\
\hline Jul 31 & P200/DBSP & $3410-5550,5750-9995$ & 600 & 1.38 \\
\hline Aug 13 & Keck II/NIRES & $9400-24650$ & 360 & 1.38 \\
\hline Aug 29 & P200/DBSP & $3410-5550,5750-9995$ & $600 \times 2$ & 1.40 \\
\hline Sep 12 & Keck II/ESI & $3950-10200$ & 1800 & 1.32 \\
\hline Sep 20 & Keck I/LRIS & $3200-10250$ & $300 \times 2$ & 1.28 \\
\hline
\end{tabular}

Note. All spectra have been uploaded to the TNS page of this source (https://www.wis-tns.org/object/2019wey). Multiple exposures were obtained on 2020 August 29 and September 20. Since no significant variability was observed on the timescale of 5-10 minutes, summed spectra were produced for the two dates.

emission features are much narrower than the $\mathrm{H} \alpha$ line, hinting at different formation locations in the accretion disk.

\section{Radio Observations}

We monitored AT2019wey with the VLA (Perley et al. 2011) under the Director's Discretionary Time programs 20A591 and 20B-397 (PI: Y. Yao). The data were calibrated using the standard VLA Pipeline. We present the flux density of our VLA detections along with the radio detections reported by Cao et al. (2020a, 2020b) in Table 2. We fit a power-law (PL) function $\left(f_{\nu} \propto \nu^{\alpha}\right)$ to the data; see Figure 9 for model fits and Table 2 for the value of $\alpha$.

Other than for August 2, the power-law fit is flat or slightly inverted $(\alpha \approx 0-0.5)$. Usually this is attributed to synchrotron self-absorption and is frequently seen in the low-hard state (LHS) and hard-intermediate state (HIMS) of X-ray binaries (Fender 2001; Fender et al. 2004). On August 2, however, a "standard" spectral index of $\sim-0.8$ was observed. The change in spectral index may indicate the existence of a multi-zone jet.
Yadlapalli et al. (2021) reported the detection of a resolved radio source by VLBA in 2020 September, which was interpreted as a steady compact jet.

\section{Discussion}

The archival (historical) optical data (see Appendix A.1) establish a faint quiescent counterpart: $r_{\text {SDSS }}>22.6$. For $0.8<$ $E(B-V)<1.2$, the corresponding extinction is $2.2<A_{r_{\mathrm{SDSs}}}<$ 3.3. Combined with our distance limit of $D<10 \mathrm{kpc}$, this restricts the donor star to have an absolute magnitude of $M_{R}>4.3$. For a main-sequence star, this corresponds to a spectral type later than $\mathrm{G} 2$ and a stellar mass $<1 M_{\odot}$. For a subgiant star, the stellar mass is even smaller. Therefore, the companion is a low-mass $\left(<1 M_{\odot}\right)$ late-type, likely evolved star. The optical outburst amplitude for AT2019wey is $\Delta r>$ $(22.6-17.4)=5.2 \mathrm{mag}$. Using an empirical relation between $\Delta r$ and $P_{\text {orb }}$ for short-period LMXBs (Shahbaz \& Kuulkers 1998), we find a orbital period, $P_{\text {orb }} \lesssim 16 \mathrm{hr}$. 


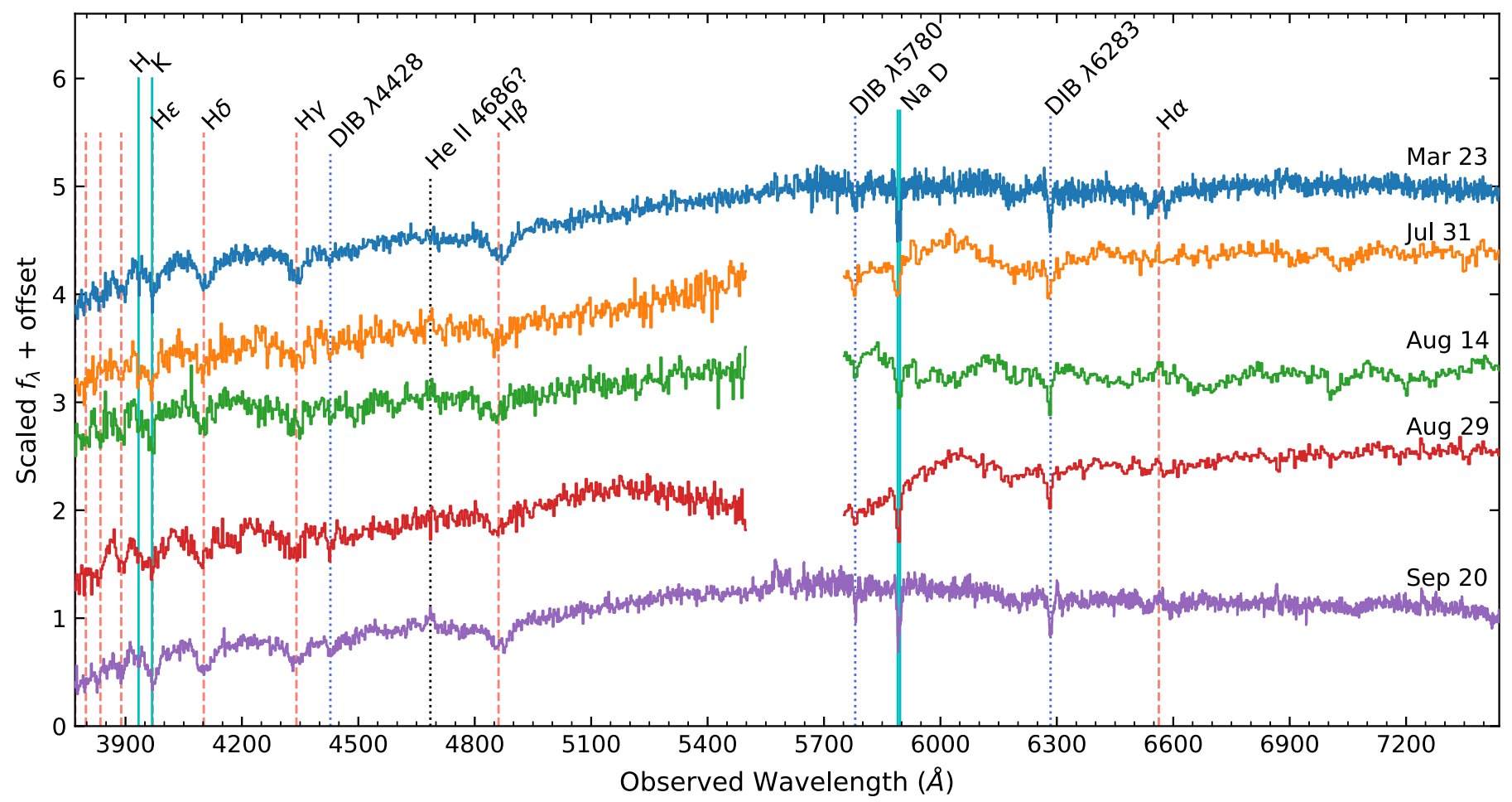

Figure 6. Low-resolution optical spectra of AT2019wey (Table 1). We note that during our DBSP observations, the blue end of the red-side CCD had a malfunction, such that flux in the 5550-5750 ̊ range was lost (masked).

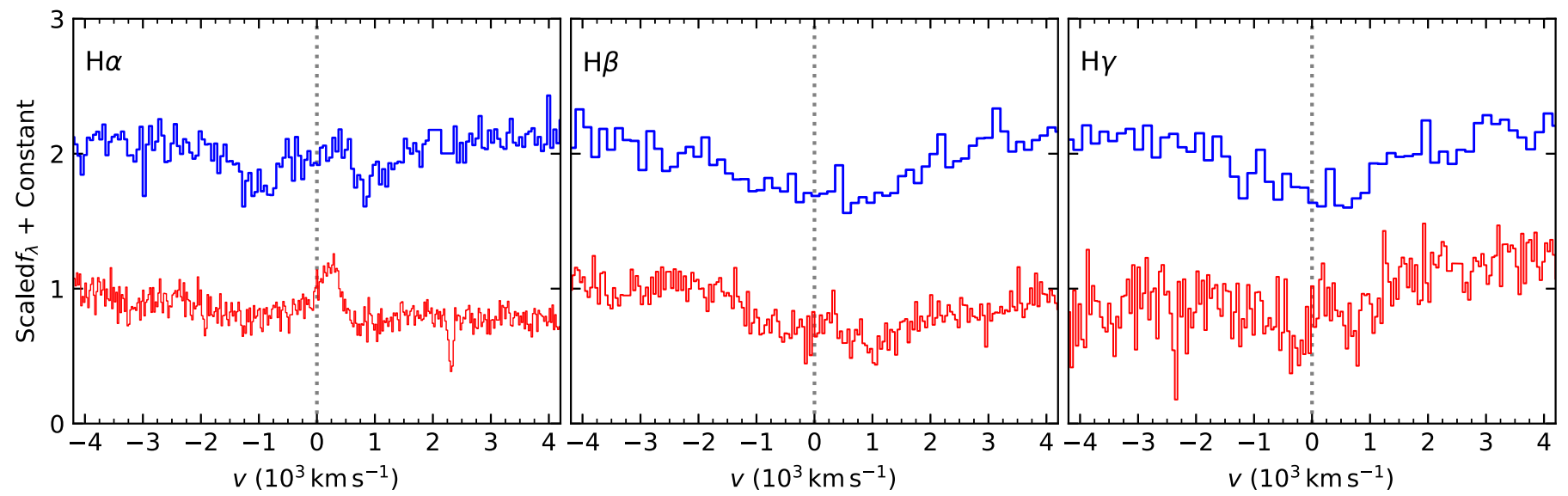

Figure 7. Velocity of the Balmer lines. The 2020 March 23 LRIS spectrum is shown on the top (in blue) and the 2020 September 12 ESI spectrum is shown on the bottom (in red).

\subsection{Radio-X-ray Correlation}

Figure 10 shows that on the $L_{\text {radio }}-L_{\mathrm{X}}$ diagram, the position of AT2019wey is above the region occupied by the majority of NS binaries and is closer to $\mathrm{BH}$ binaries. Therefore, the bright radio luminosity favors a $\mathrm{BH}$ accretor.

\subsection{Multi-wavelength Light Curve}

We separate the multi-wavelength light curve of AT2019wey into five stages (see the bottom panel of Figure 2): (i) before MJD 58814, the source was in quiescence, (ii) from MJD 58814 to MJD $\sim 58880$, the optical light curve exhibited a fast-rise linear-decay outburst, after which it settled onto a $r$-band flux of $f_{\nu, r} \sim 315 \mu \mathrm{J} y$; around the same time, the X-ray flux rose to $\sim 1 \mathrm{mCrab}$, and stayed in the LHS, (iii) from MJD $\sim 58880$ to MJD 59010, the optical and X-ray light curves stayed almost flat, (iv) From MJD 59010 to MJD 59080, AT2019wey exhibited a multi-wavelength brightening and the X-ray remained in the LHS (Paper I), (v) from MJD 59081 to MJD 59180, the source entered into the HIMS (Paper I). The optical stayed around $f_{\nu, r} \sim 400 \mu J y$ and X-ray stayed around $\sim 20 \mathrm{mCrab}$ (Paper I).

\subsubsection{UV/optical-X-ray Correlation}

During stage (iv), the X-ray and radio fluxes increased by a factor of $\gtrsim 10$ but in the optical/UV the increase was modest, between a factor of 1.3 and 2. During stages (iii) and (v), the source was stable and representative luminosities can be found in Table 3. For these two stages, following Russell et al. (2006), we link the UV/optical and X-ray luminosities as

$$
L_{\mathrm{UV} / \mathrm{opt}}=A L_{\mathrm{X}}^{\beta},
$$




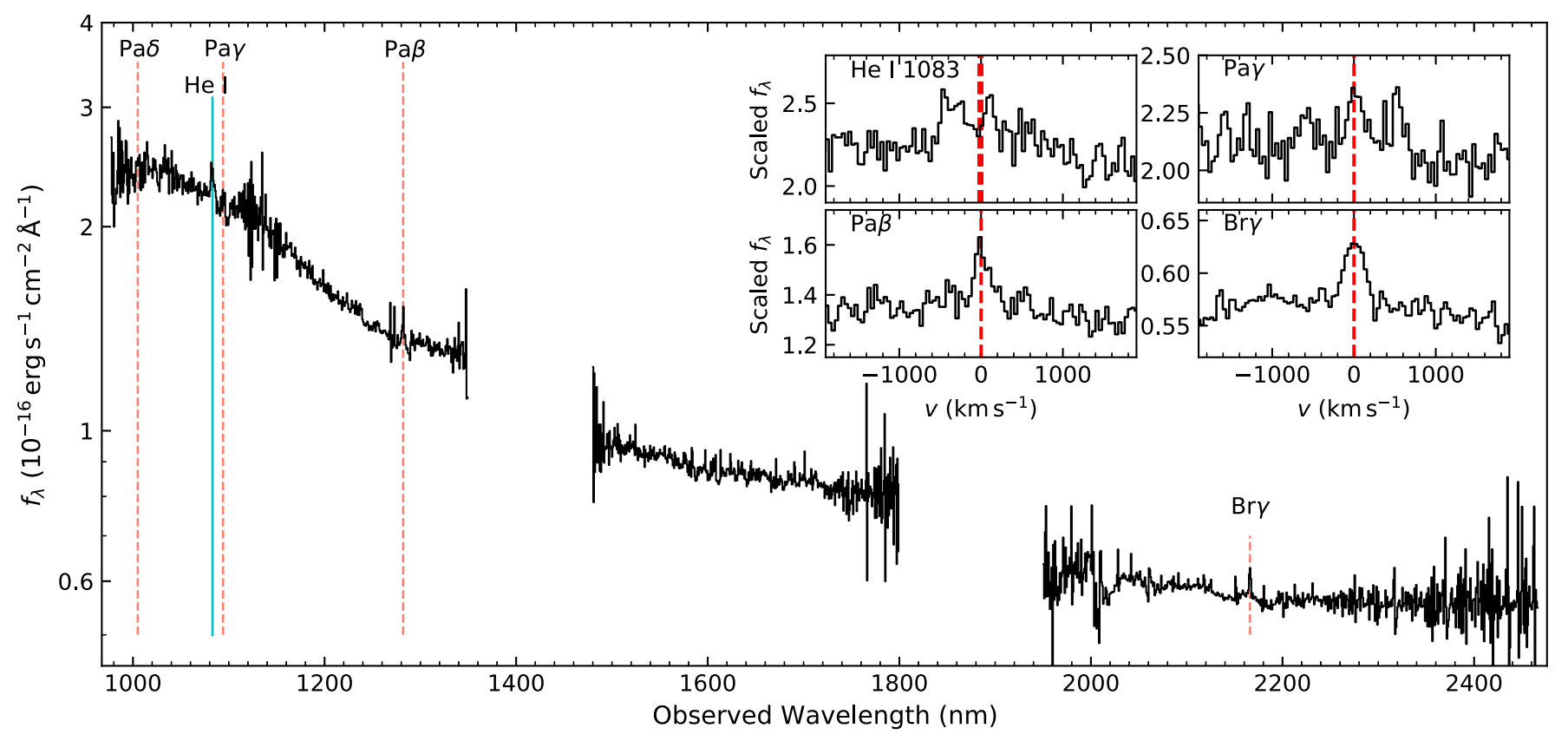

Figure 8. NIRES spectrum of AT2019wey. The insets show the zoom-in on emission lines in velocity space.

and find $\beta \sim 0.08$ in $r$ band, $\beta \sim 0.12$ in $g$ band, and $0.12 \lesssim \beta \lesssim 0.34$ in the UV bands. Russell et al. (2006) derived $A=10^{13.1^{ \pm 0.6}}, \beta=0.61 \pm 0.02$ for a sample of $15 \mathrm{BH}$ LMXBs and $A=10^{10.8 \pm 1.4}, \beta=0.63 \pm 0.04$ for a sample of $8 \mathrm{NS}$ LMXBs. As can be seen from Figure 11, over the distance range of $1 \lesssim D \lesssim 10 \mathrm{kpc}$, the inferred luminosities of AT2019wey are suggestive of an accreting $\mathrm{BH}$ system.

\subsubsection{Possible Mechanisms for the Optical Emission}

In BH LMXBs in the hard state, the optical/UV emission can arise from (1) X-ray reprocessing in the outer accretion disk, (2) the optically thick jet spectrum extending from centimeter wavelengths, or (3) intrinsic thermal emission from the viscously heated outer accretion disk. For processes (1) and (2), the expected slopes are $\beta \sim 0.5$ (van Paradijs \& McClintock 1994), and $\beta \sim 0.7$ (Corbel et al. 2003; Russell et al. 2006), respectively. For process (3), $\beta$ ranges from 0.13 (Rayleigh-Jeans or R-J tail) to 0.33 (between the $\mathrm{R}-\mathrm{J}$ tail and the Wien cutoff) (Tetarenko et al. 2020). Russell et al. (2006) find $\beta \sim 0.6$ for BH LMXBs, which suggests that process (3) is not dominant. However, the observed $\beta \sim 0.1$ for AT2019wey favors process (3).

Curiously, we note that such small values of $\beta$ have been observed in two BH LMXBs with short orbital periods: $\beta \sim 0.2$ (Armas Padilla et al. 2013) in Swift J1357.2-0933 ( $P_{\text {orb }}=2.8 \mathrm{~h}$; Corral-Santana et al. 2013; Mata Sánchez et al. 2015) and $\beta \sim 0.2$ (Chiang et al. 2010) in Swift J1753.5-0127 ( $P_{\text {orb }} \lesssim 3.2 \mathrm{~h}$; Zurita et al. 2008; Neustroev et al. 2014; Shaw et al. 2016). Interestingly, the X-rays for these two systems are only observed in the LHS or HIMS, without successful transitions to the high/soft state (HSS) (Armas Padilla et al. 2013; Tetarenko et al. 2016). These similarities might be understood as characteristics of a subpopulation of BH LMXBs (Shaw et al. 2013, see Section 7).

\subsection{Multi-wavelength SED}

The spectral energy distribution (SED) of AT2019wey is shown in Figure 12. The X-ray data are presented in Paper I and we briefly summarize the X-ray spectra in Section 6.3.1. In Section 6.3.2, based on radio data, we conclude that jet emission is unlikely to be the dominant mechanism in the optical. In Section 6.3.3, we show that the UV/optical emission during stage (iii) originates from the intrinsic emission of a truncated accretion disk. In Section 6.3.4, we show that the $\mathrm{UV} /$ optical emission during stage (v) arises from X-ray reprocessing.

\subsubsection{The X-ray SED}

Briefly speaking, the X-ray spectrum observed in stage (iii) can be described by an absorbed power-law with photon index $\Gamma=1.8$. In stages (iv) and (v), the X-ray spectrum can be fitted with a combination of disk-blackbody (diskbb, Shakura \& Sunyaev 1973; Mitsuda et al. 1984) and power-law components (Paper I). On August 14, 21, and 28, the fitted models have $\Gamma \sim 1.9$ and inner-disk temperature $T_{\text {disk }} \sim 0.21 \mathrm{keV} \sim 2.4 \times$ $10^{6} \mathrm{~K}$. The inner-disk radius is

$$
R_{\text {in }} \sim(360-470)\left(\frac{\cos i}{1}\right)^{-1 / 2}\left(\frac{D}{5 \mathrm{kpc}}\right) \mathrm{km} .
$$

On September 20, the soft X-ray flux reached a local maximum in the HIMS, where the PL softened to $\Gamma=2.3$ and the innerdisk temperature increased to $T_{\text {disk }} \sim 0.29 \mathrm{keV} \sim 3.4 \times 10^{6} \mathrm{~K}$, while the inner-disk radius remains at $\sim 400 \mathrm{~km}$. The fitted $T_{\text {disk }}$ and $R_{\text {in }}$ are typical for thermal emission from a truncated accretion disk observed in the LHS and HIMS of BH LMXBs (Done et al. 2007). Denoting the innermost stable circular orbit radius as $R_{\mathrm{ISCO}}=6 G M / c^{2}$ and the Schwarzschild radius as $R_{\mathrm{S}}=2 G M / c^{2}$, then $R_{\text {in }} \sim 15 R_{\mathrm{S}} \sim 5 R_{\mathrm{ISCO}}$ for a $10 M_{\odot}$ nonspinning black hole.

\subsubsection{The Radio SED}

The dashed-dotted lines shown in Figure 12 are best-fit power-law modes for the radio data (Table 2) extrapolated to 
Table 2

Radio Observations of AT2019wey

\begin{tabular}{|c|c|c|c|}
\hline Date & $\nu_{0}(\mathrm{GHz})$ & $f_{\nu}(\mu \mathrm{Jy})$ & $\alpha$ \\
\hline $2020-05-27$ & $\begin{array}{l}5.0 \\
6.0 \\
7.0\end{array}$ & $\begin{array}{l}197 \pm 20 \\
220 \pm 22 \\
234 \pm 23\end{array}$ & $0.51 \pm 0.69$ \\
\hline 2020-08-02 & $\begin{array}{c}2.5 \\
3.5 \\
10.0\end{array}$ & $\begin{array}{c}218 \pm 49 \\
205 \pm 16 \\
82 \pm 11\end{array}$ & $-0.82 \pm 0.23$ \\
\hline 2020-08-14 & $\begin{array}{c}1.5 \\
2.5 \\
3.5 \\
8.5 \\
9.5 \\
10.5 \\
11.5\end{array}$ & $\begin{array}{c}1023 \pm 75 \\
998 \pm 59 \\
1077 \pm 18 \\
1420 \pm 12 \\
1399 \pm 11 \\
1447 \pm 13 \\
1431 \pm 13\end{array}$ & $0.23 \pm 0.02$ \\
\hline $2020-08-21$ & $\begin{array}{c}1.5 \\
2.5 \\
3.5 \\
8.5 \\
9.5 \\
10.5 \\
11.5\end{array}$ & $\begin{array}{c}1676 \pm 102 \\
1767 \pm 51 \\
1923 \pm 18 \\
2340 \pm 18 \\
2393 \pm 18 \\
2376 \pm 18 \\
2353 \pm 19\end{array}$ & $0.19 \pm 0.01$ \\
\hline $2020-08-28$ & $\begin{array}{c}1.5 \\
2.5 \\
3.5 \\
8.5 \\
9.5 \\
10.5 \\
11.5\end{array}$ & $\begin{array}{c}1846 \pm 128 \\
1891 \pm 34 \\
2048 \pm 15 \\
2529 \pm 11 \\
2542 \pm 16 \\
2536 \pm 18 \\
2511 \pm 20\end{array}$ & $0.20 \pm 0.01$ \\
\hline $2020-10-17$ & 6.7 & $1350 \pm 220$ & $\cdots$ \\
\hline 2021-02-17 & $\begin{array}{c}1.5 \\
2.5 \\
3.5 \\
8.5 \\
9.5 \\
10.5 \\
11.5\end{array}$ & $\begin{array}{l}1565 \pm 44 \\
1394 \pm 16 \\
1435 \pm 10 \\
1658 \pm 12 \\
1553 \pm 13 \\
1407 \pm 11 \\
1295 \pm 11\end{array}$ & $0.01 \pm 0.01$ \\
\hline
\end{tabular}

Note. $\nu_{0}$ is central frequency. The spectral index $\alpha\left(f_{\nu} \propto \nu^{\alpha}\right)$ is fitted using the Markov Chain Monte Carlo (MCMC) approach with emcee (ForemanMackey et al. 2013). The uncertainties are calculated using the $90 \%$ quantiles from the MCMC run

$3 \times 10^{12} \mathrm{~Hz}$. If the spectrum remains optically thick all the way to the optical and near-infrared (OIR) wavelengths, it will overpredict the observed OIR spectrum. Assuming a classical jet spectrum of a broken PL (Blandford \& Königl 1979), the break frequency must be $\ll 10^{14} \mathrm{~Hz}$. The optically thin jet spectrum may contribute a fraction of NIR emission (gray data in Figure 12), but is unlikely to dominate in the optical.

\subsubsection{UV/Optical Emission in the Dim LHS}

In Figure 13, we show the UV/optical data and the best-fit $\mathrm{X}$-ray model in the dim LHS (stage iii) in orange. The low level of X-ray flux (compared to that in the UV/optical) suggests that there is not enough X-ray flux to illuminate the outer accretion disk. As a result, the UV/optical probably comes from the intrinsic thermal emission of an accretion disk.

To obtain a constraint on the outermost annulus of the accretion disk, we compute a set of simple blackbody models (upper panel

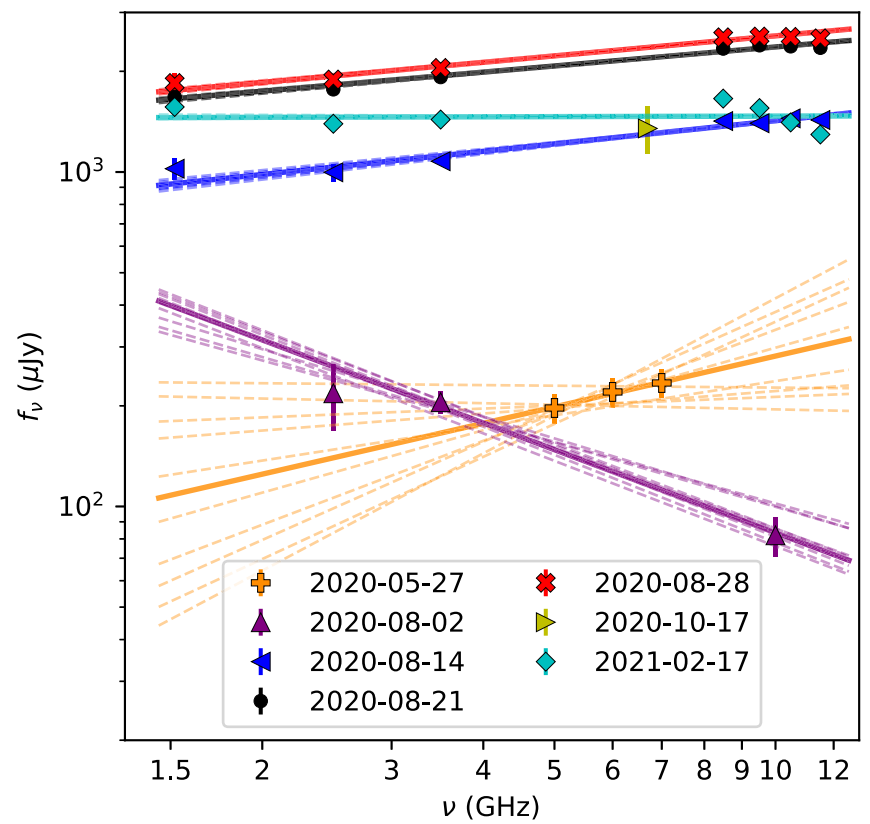

Figure 9. Radio observations of AT2019wey. The solid lines are model fits using estimated parameters. Ten random draws from the MCMC posterior are shown with dashed lines. Note that the random draws for the well-constrained models are so well aligned that they cannot be seen.

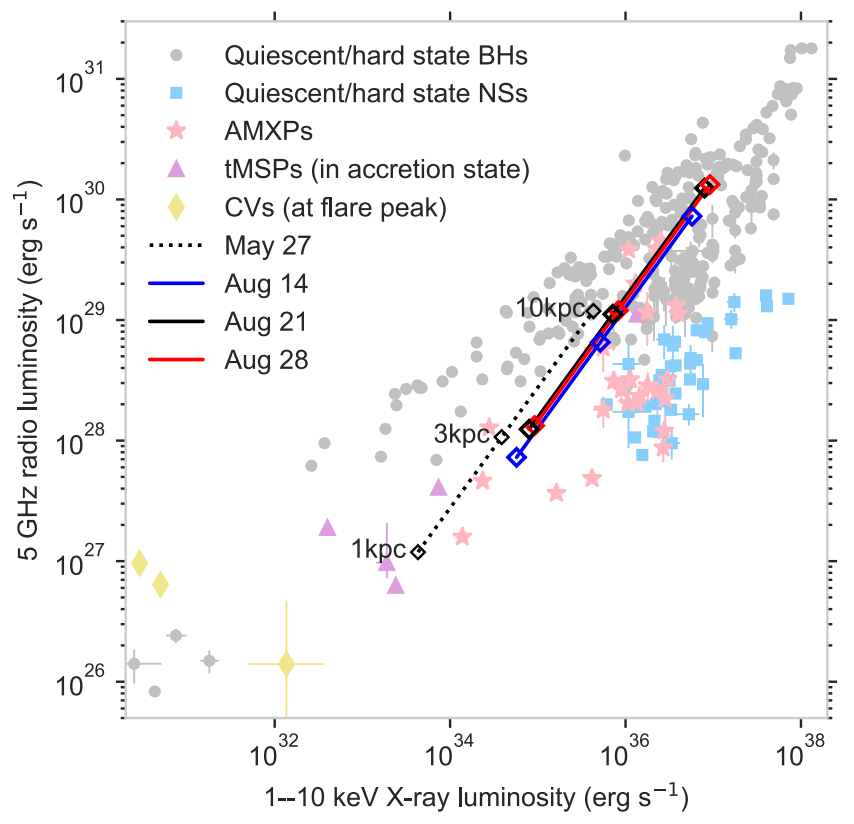

Figure 10. The $L_{\mathrm{radio}}-L_{\mathrm{X}}$ diagram of AT2019wey and various populations of $\mathrm{X}$-ray sources, including quiescent/hard state BHs, NSs, accreting millisecond $\mathrm{X}$-ray pulsars (AMXPs), transitional millisecond pulsars (tMSPs), and cataclysmic variables (CVs) (Bahramian et al. 2018). We mark the positions of AT2019wey at four epochs for possible distances of 1-3-10 kpc.

of Figure 13). We adopt the $11,000 \mathrm{~K}$ blackbody as an approximation of the outer disk annulus and compute a set of diskbb models to obtain a lower limit to the inner-disk radius (and an upper limit to the inner-disk temperature). The dotted line in the lower panel of Figure 13 suggests $T_{\text {in }}<4.8 \times 10^{5} \mathrm{~K}$ and $R_{\text {in }}>3.3 \times 10^{3} \mathrm{~km} \sim 38 R_{\mathrm{ISCO}} \sim 114 R_{\mathrm{S}}$.

Similar SED shapes have been observed in the LHS of a few BH LMXBs, including XTE J1118+480 $\left(R_{\text {in }}=300 R_{\mathrm{S}}\right.$; 
Table 3

X-ray and Optical Luminosity of AT2019wey at Different Stages of the Multi-wavelength Evolution

\begin{tabular}{lccc}
\hline \hline Stage & Band & Luminosity & Comments \\
\hline (iii) & $r$ and $g$ & $4.0 \times 10^{34} \& 6.1 \times 10^{34}$ & Averaged between MJD $\sim 58880$ and MJD $\sim 59010$ \\
(iii) & X-ray & $1.0 \times 10^{35}$ & Averaged between MJD $\sim 58951$ and MJD $\sim 58967$ \\
\hline (v) & $r$ and $g$ & $4.9 \times 10^{34} \& 8.4 \times 10^{34}$ & Averaged between MJD $\sim 59080$ and MJD $\sim 59153$ \\
(v) & X-ray & $(1.3-1.7) \times 10^{36}$ & Range of values from minimum (MJD 59082) to maximum (MJD $\sim 59112)$ \\
\hline
\end{tabular}

Note. Luminosity is given in units of $(D / 5 \mathrm{kpc})^{2} \mathrm{erg} \mathrm{s}^{-1}$. X-ray column density corrected luminosity is given in $2-10 \mathrm{keV}$, assuming $N_{\mathrm{H}}=5 \times 10^{21} \mathrm{~cm}^{-2}$. Optical luminosity has been corrected for extinction, adopting $E(B-V)=0.9$.

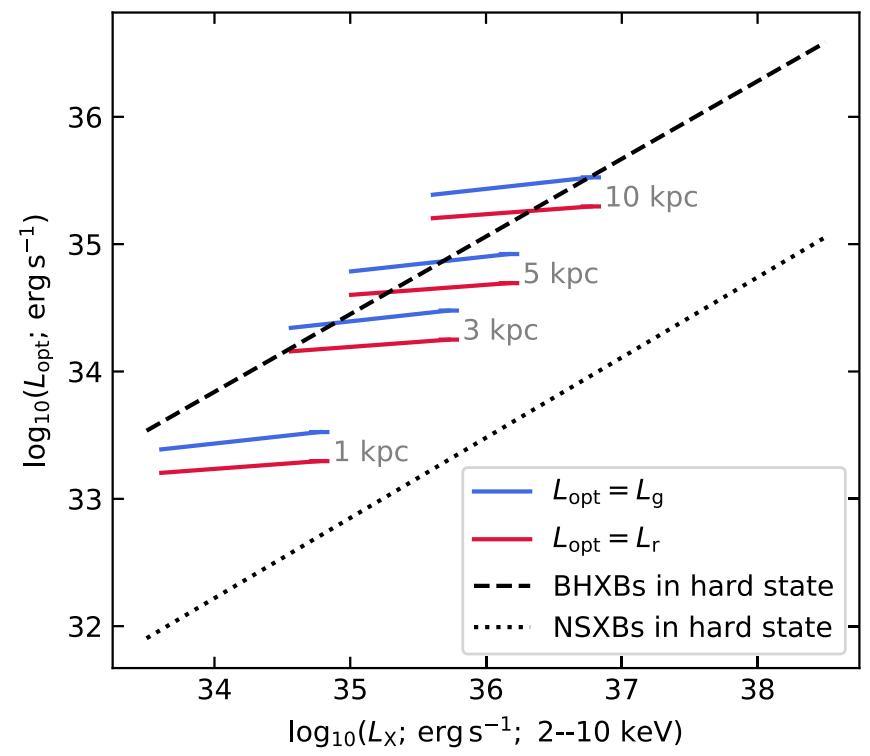

Figure 11. The solid lines demonstrate the correlation between optical ( $g$ - or $r$ band) and X-ray luminosities of AT2019wey from stage (iii) to stage (v), assuming distances at $[1,3,5,10] \mathrm{kpc}$. The dashed and dotted lines are best power-law fits to BH X-ray binaries (BHXBs) and NS X-ray binaries (NSXBs) in the hard state, respectively (Russell et al. 2006).

Yuan et al. 2005) and Swift J1753.5-0127 $\left(R_{\text {in }}>100 R_{\text {S }}\right.$; Froning et al. 2014). The observed SED of AT2019wey in the dim LHS fits into the advection-dominated accretion flow (ADAF; Narayan \& Yi 1994, 1995) model of a hot accretion flow around a BH, which is predicted at low accretion rates (see reviews by Done et al. 2007; Yuan \& Narayan 2014; Poutanen \& Veledina 2014). If so, the X-ray PL comes from a hightemperature flow in the central regions close to the $\mathrm{BH}$, while the UV/optical thermal component comes from a geometrically thin, optically thick accretion disk truncated far from the ISCO (Yuan \& Narayan 2014).

\subsubsection{UV/Optical Emission in the HIMS}

The dotted green line in Figure 12 shows an extrapolation of the diskbb fit on NICER data for September 20. It clearly under-predicts the observed UV/optical spectrum, making $\mathrm{X}$-ray reprocessing the most likely origin of the UV/optical emission in the HIMS. We therefore attempt to fit the green data by the irradiation model diskir (Gierliński et al. 2008, 2009).

We set the inner-disk temperature of the un-illuminated disk and the asymptotic PL photon index to be the same as the best-fit September 20 model (see Section 6.3.1). The fraction of reprocessed luminosity in the Compton tail $\left(f_{\text {in }}\right)$ is fixed at 0.1 . The electron temperature is fixed at $1000 \mathrm{keV}$ as there is no sign of a high-energy PL cutoff (see Paper I). The dashed green line in Figure 12 is a schematic fit with the following parameters: the ratio of luminosity in the Compton tail to that of the unilluminated disk $L_{\mathrm{C}} / L_{\mathrm{d}}=0.22$, the radius of the Comptonilluminated disk $R_{\mathrm{irr}}=1.2 R_{\mathrm{in}}$, the fraction of thermalized bolometric flux $f_{\text {out }}=0.08, R_{\text {out }}=10^{3.55} R_{\text {in }}$, and the normalization parameter of the un-illuminated disk (Equation (2)) $\approx 370 \mathrm{~km}$. We conclude that the UV/optical SED in the HIMS is due to reprocessing of the X-ray irradiation.

\subsection{Optical Spectral Lines}

The hydrogen lines in AT2019wey display both broad absorption and emission components (Section 4.1). This behavior is reminiscent of some LMXBs and CVs, where the hydrogen absorption and emission lines are thought to arise from different layers of the viscous accretion disk (Horne \& Marsh 1986; La Dous 1989; Warner 1995). In a few BH LMXBs, such as GRO J1655-40 (Soria et al. 2000), GRO J0422+32 (Callanan et al. 1995), XTEJ1118+480 (Dubus et al. 2001; Torres et al. 2002), and Swift J1753.5-0127 (Rahoui et al. 2015), doublepeaked $\mathrm{H} \alpha$ was observed. The single-peaked hydrogen line profile of AT2019wey is similar to that observed in MAXI J1836 -194 (Russell et al. 2014), suggesting a binary system viewed close to face-on. This is in agreement with the low inclination $\left(i \lesssim 30^{\circ}\right)$ constraint from modeling the X-ray reflection spectrum (Paper I).

In Section 6.3.4 we have shown that in the HIMS, the UV/ optical emission comes from the reprocessing of inner-disk and coronal emission. Irradiation of the outer disk may form a thin temperature-inversion layer on the disk surface (Tuchman et al. 1990). This naturally explains the enhanced Balmer emission lines observed during stage (iv) and stage (v).

Most BH LMXBs show strong He II emission during their outbursts (Zurita et al. 2002; Kaur et al. 2012; Jiménez-Ibarra et al. 2019; Russell et al. 2014). A lack of significant He II was observed in the optical spectra of AT2019wey. This might also be present in the 2009 outburst of XTE J1752-223 (Torres et al. 2009) and the 2021 outburst of XTE J1859+226 (Bellm 2021, Bellm, E. C. et al., 2021 in preparation). We note that the He II recombination line was also not significantly detected in the outburst spectra of a few CVs (Morales-Rueda \& Marsh 2002). A possible explanation for this is that the number of photons with energies between $54 \mathrm{eV}$ (the ionization potential of $\mathrm{He}^{+}$) and $280 \mathrm{eV}$ (the ionization potential of the carbon K-edge) is not large enough (Patterson \& Raymond 1985). 


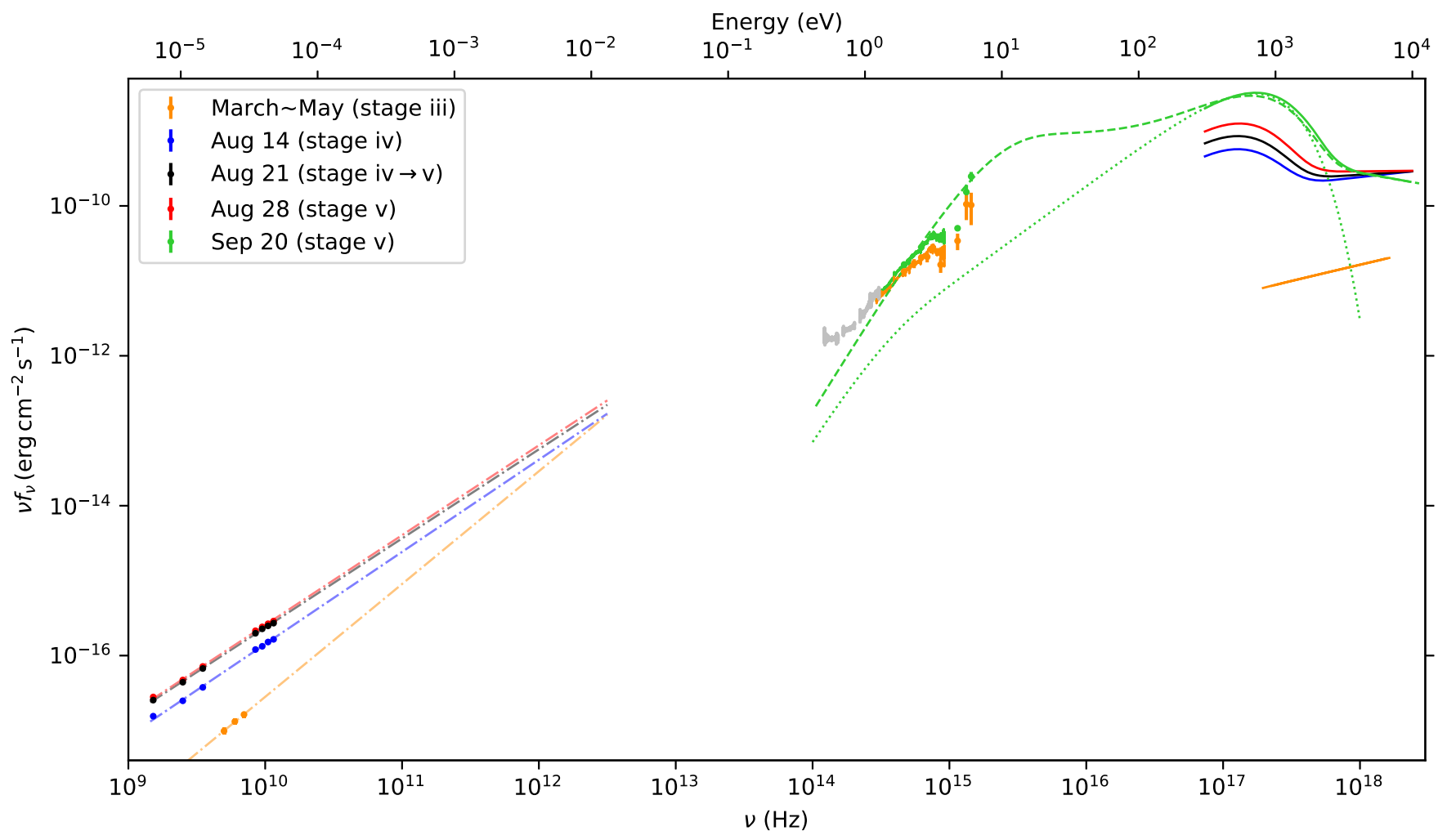

Figure 12. Multi-wavelength SED of AT2019wey. In the radio, we show the observed data and power-law fits (Table 2). In UV/optical/NIR, we show the dereddened photometry and spectra assuming $E(B-V)=0.9$. Note that the silver NIR spectrum, the orange optical spectrum, and the green optical spectrum were obtained on August 13, March 20, and September 20, respectively (Table 1). In X-ray, we show the best fits to X-ray data corrected for a fixed column density of $N_{\mathrm{H}}=5 \times 10^{21} \mathrm{~cm}^{-2}$ (see Section 6.3.1 and Paper I). See definition of different stages in Section 6.2. The dashed and dotted green lines from optical to X-ray are illustrative models of irradiation and standard disk emission, respectively (see Section 6.3.4 for details).

\section{Conclusion}

We have undertaken a detailed multi-wavelength follow up of the X-ray transient AT2019wey. This study builds upon $\mathrm{X}$-ray observations reported in Paper I, which show that AT2019wey is an LMXB with an NS or BH accretor. In this paper, we present the high radio (Section 6.1) and optical (Section 6.2.1) luminosities of AT2019wey. These properties, combined with the hard X-ray spectrum reported in Paper I, indicate that AT2019wey is likely a BH system.

Multi-wavelength evolution of AT2019wey can be separated into five distinct stages, as illustrated in Figure 2. In the dim LHS [i.e., stage (iii)], the UV/optical emission comes from intrinsic thermal emission of an accretion disk with $R_{\text {in }}>$

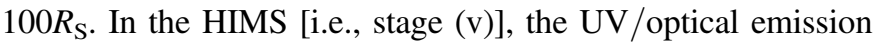
comes from reprocessing of $\mathrm{X}$-rays and the disk truncation radius has moved inward $\left(R_{\mathrm{in}} \sim 15 R_{\mathrm{S}}\right)$. The overall SED evolution fits into the picture of a hot accretion flow consisting of an inner ADAF and a truncated disk. This confirms the widely accepted model for short-period BH LMXBs in the hard state.

The optical light curve of AT2019wey is distinguished by its flatness during stages (iii) and (v). This is different from the majority of LMXBs and is similar to what was observed during the $12 \mathrm{yr}$ outburst of Swift J1753.5-0127 (Shaw et al. 2019; Zhang et al. 2019). The $\mathrm{X}$-ray light curve is reminiscent of the "flat-top" profile in the 1996 outburst of GRO J1655-40 (Esin et al. 2000). As noted before (Esin et al. 2000; Shaw et al. 2019), the "standstill" outburst is analogous to the Z Cam class of dwarf novae (Osaki 1996). In such systems, the masstransfer rate $\left(\dot{M}_{2}\right)$ during quiescence is $\lesssim \dot{M}_{\text {crit }}$. Here $\dot{M}_{\text {crit }}$ is the critical mass-transfer rate, above which the disk remains stable (Dubus et al. 1999; Lasota et al. 2008). During the outburst, $\dot{M}_{2}$ increased to $\gtrsim \dot{M}_{\text {crit }}$, stabilizing the accretion. In AT2019wey, the second stable period in stage (v) indicates a further increase of $\dot{M}_{2}$, probably caused by irradiation on the accretion disk or the companion star.

We note that if AT2019wey continues to remain sufficiently bright in the optical for an extended period of time, the next data release of the Gaia mission may help to further constrain the distance. Once the distance is settled, future studies can estimate $\dot{M}_{2}$ during the stable stages. Comparison between $\dot{M}_{2}$ and $\dot{M}_{\text {crit }}$ can provide a key probe to the evolution of X-ray binaries.

As discussed in Paper I, SRG is sensitive to the population of BH LMXBs with faint X-ray outbursts. These outbursts are generally associated with lower mass accretion rates and shorter orbital periods (Meyer-Hofmeister 2004; Wu et al. 2010; Tetarenko et al. 2016). The discovery of AT2019wey showcases the possibility of hunting for similar systems in wide-field optical surveys. This has also been demonstrated in the case of the BH LMXB ASASSN-18ey (MAXIJ1820 +070 ), which was first discovered in the optical (Tucker et al. 2018) and then in the X-ray (Kawamuro et al. 2018). Perhaps the easiest approach to identify similar LMXBs is to study optical light curves of SRG point sources in the Galactic plane. 


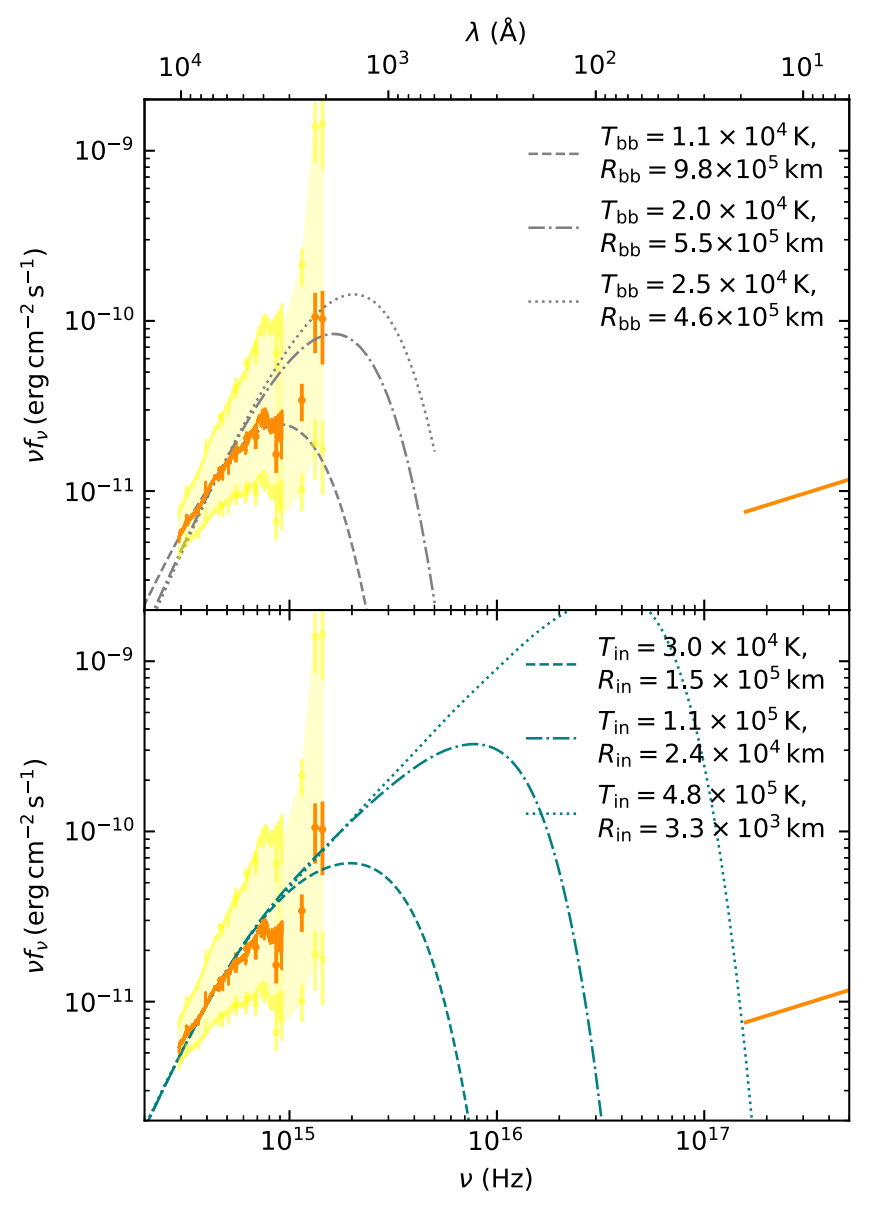

Figure 13. X-ray-UV-optical SED of AT2019wey in the dim LHS. Singletemperature blackbody models are shown in the upper panel, while diskblackbody models are shown in the lower panel. All models are normalized to match the flux in $r$ band. $D=5 \mathrm{kpc}$ and $\cos i=1$ are assumed. The upper and lower bounds of the yellow region are obtained by dereddening the observed data using $E(B-V)=1.2$ and 0.7 , respectively. No detailed model fits are performed due to the uncertainty of $E(B-V)$.

We thank the anonymous reviewer for providing comments that have significantly improved this manuscript. We thank Mark McKinnon and Amy Mioduszewski for allocating DD time on VLA. We thank Jie Lin and Stephen Smartt for helpful comments.

Y.Y. is supported in part by the Heising-Simons Foundation. M.M.K. acknowledges generous support from the David and Lucille Packard Foundation. E.C.B. is supported in part by the NSF AAG grant 1812779 and grant \#2018-0908 from the Heising-Simons Foundation.

This work is based on observations obtained with the 48inch Samuel Oschin Telescope and the 60-nch Telescope at the Palomar Observatory as part of the Zwicky Transient Facility project. The ZTF is supported by the National Science Foundation under grant No. AST-1440341 and a collaboration including Caltech, IPAC, the Weizmann Institute for Science, the Oskar Klein Center at Stockholm University, the University of Maryland, the University of Washington, Deutsches Elektronen-Synchrotron and Humboldt University, Los Alamos National Laboratories, the TANGO Consortium of Taiwan, the University of Wisconsin at Milwaukee, and Lawrence Berkeley National Laboratories. Operations are conducted by COO, IPAC, and UW.
SED Machine is based upon work supported by the National Science Foundation under grant No. 1106171. This work was supported by the GROWTH project funded by the National Science Foundation under grant No. 1545949. The ZTF forcedphotometry service was funded under the Heising-Simons Foundation grant \#12540303 (PI: Graham).

This work has made use of data from the Asteroid Terrestrial-impact Last Alert System (ATLAS) project. The Asteroid Terrestrial-impact Last Alert System (ATLAS) project is primarily funded to search for near-Earth asteroids through NASA grants NN12AR55G, 80NSSC18K0284, and 80NSSC18K1575; byproducts of the NEO search include images and catalogs from the survey area. This work was partially funded by Kepler/K2 grant J1944/80NSSC19K0112 and HST GO-15889, and STFC grants ST/T000198/1 and ST/S006109/1. The ATLAS science products have been made possible through the contributions of the University of Hawaii Institute for Astronomy, the Queens University Belfast, the Space Telescope Science Institute, the South African Astronomical Observatory, and The Millennium Institute of Astrophysics (MAS), Chile.

We acknowledge ESA Gaia, DPAC, and the Photometric Science Alerts Team (http://gsaweb.ast.cam.ac.uk/alerts).

Facilities: PO:1.2 m (ZTF, iPTF, POSS), PO:1.5 m (SEDM), Gaia, Hale (DBSP, CHIMERA), Keck:I (LRIS), Keck:II (ESI, NIRES), VLA, MAXI, Swift (UVOT, XRT), NICER, Sloan, PS1.

Software:astropy (Astropy Collaboration et al. 2013), CASA (v5.6.1; McMullin et al. 2007), diskir (Gierliński et al. 2008, 2009), emcee (Foreman-Mackey et al. 2013), FPipe (Fremling et al. 2016), HEASoft (v6.27; Heasarc 2014), LPipe (Perley 2019), makee (https://sites.astro. caltech.edu/tb/ipac_staff/tab/makee/), matplotlib (Hunter 2007), pandas (McKinney 2010), pyraf-dbsp pipeline (Bellm \& Sesar 2016), scipy (Virtanen et al. 2020), spextool (Cushing et al. 2004), XRB-LrLx_pub (Bahramian et al. 2018, https://github.com/bersavosh/XRB-LrLx_pub), xspec (v12.11.0; Arnaud 1996) xtellcor (Vacca et al. 2003).

\section{Appendix A Archival Limits}

\section{A.1. Optical Limits}

We conducted an archival search of optical photometry at the position of AT2019wey. The source was not detected by historical optical surveys, including the Palomar Observatory Sky Survey I (POSS-I, Minkowski \& Abell 1963), the Second Palomar Observatory Sky Survey (POSS-II, Reid et al. 1991), SDSS, and the Panoramic Survey Telescope and Rapid Response System (Pan-STARRS, PS1) DR1 (Flewelling et al. 2020; Waters et al. 2020), the intermediate Palomar Transient Factory (iPTF; Law et al. 2009; Rau et al. 2009), and the ZTF. We list $5 \sigma$ upper limits in Table 4.

\section{A.2. Radio Limit}

AT2019wey was not detected in any archival radio database. The NRAO VLA Sky Survey (NVSS, Condon et al. 1998) provides an upper limit of $2 \mathrm{mJy}$ at $1.4 \mathrm{GHz}$ in 1993-1996. The Karl G. Jansky Very Large Array Sky Survey (VLASS, Lacy et al. 2020) provides a $3 \sigma$ upper limit of $0.40 \mathrm{mJy}$ at $2-4 \mathrm{GHz}$ in March 2019. 
Table 4

Historical Upper Limits at the Position of AT2019wey

\begin{tabular}{lcccc}
\hline \hline Survey & Time & Filter & $\lambda_{\text {eff }}(\AA)$ & Limit \\
\hline POSS-I & $1953-10-08$ & $r$ & 6500 & 19.5 \\
POSS-II & $1990-10-26$ & $r$ & 6500 & 20.8 \\
\hline SDSS & $2004-10-15$ & $u$ & 3560 & 22.5 \\
& & $g$ & 4720 & 23.1 \\
& & $r$ & 6190 & 22.6 \\
& & $i$ & 7500 & 22.0 \\
& & $z$ & 8960 & 20.9 \\
\hline PS1 & & $g$ & 4870 & 22.7 \\
& & $r$ & 6210 & 22.3 \\
& & $i$ & 7540 & 22.1 \\
& & $z$ & 8680 & 21.8 \\
\hline iPTF & $2010-02-2014-12$ & $y$ & 9630 & 20.8 \\
\hline ZTF & $2017-12-2019-11$ & $g$ & 6420 & 21.0 \\
& & $r$ & 4810 & 21.3 \\
& & & 6420 & 21.5 \\
\hline
\end{tabular}

\section{Appendix B \\ Instrumental/Observational Information}

We provide ZTF photometry in Table 5. UVOT and SEDM photometry is provided in Table 6 .

We obtained optical spectroscopic follow-up observations of AT2019wey using the Low-Resolution $(R \approx 1000)$ Imaging Spectrograph (LRIS; Oke et al. 1995) on the Keck I telescope, the Double Spectrograph (DBSP; $R \approx 1200$; Oke \& Gunn 1982) on the 200-inch Hale telescope, and the medium-resolution $(R \approx 13,000)$ Echellette Spectrograph and Imager (ESI; Sheinis et al. 2002) on the Keck II telescope. We obtained NIR spectroscopy using the Near-Infrared Emission Spectroscopy (NIRES; $R=2700$ ) on the Keck II telescope. Spectroscopic observations were coordinated with the GROWTH Marshal (Kasliwal et al. 2019).

The DBSP spectra were reduced using the pyraf-dbsp pipeline (Bellm \& Sesar 2016). The LRIS spectra were reduced and extracted using Lpipe (Perley 2019). The flat-fielding, wavelength solution (using sky lines), and extraction for the NIRES spectrum was carried out using the spextool code (Cushing et al. 2004). The extracted spectrum was fluxcalibrated using the telluric A0V standard star HIP 16652 with the xtellcor code (Vacca et al. 2003). The ESI spectrum was reduced using the $\mathrm{MAKEE}^{17}$ pipeline following the standard procedure. Flux calibration was not performed on the ESI spectrum.
Table 5

ZTF Forced Photometry of AT2019wey

\begin{tabular}{lccc}
\hline \hline MJD & $f_{\nu}(\mu \mathrm{Jy})$ & $\sigma_{f_{\nu}}(\mu \mathrm{Jy})$ & Filter \\
\hline 58206.1662 & -12.13 & 7.82 & $g$ \\
58207.1664 & 0.03 & 9.18 & $g$ \\
58210.2064 & 3.97 & 12.89 & $g$ \\
58218.2068 & 3.30 & 7.63 & $r$ \\
58219.1712 & -2.47 & 10.48 & $r$ \\
58231.1454 & -11.46 & 7.32 & $r$ \\
58234.1575 & -7.03 & 9.70 & $g$ \\
58236.1591 & -0.51 & 12.99 & $g$ \\
\hline
\end{tabular}

Note. Data up to 2020 November 30 are included. $f_{\nu}$ is observed flux density (without extinction correction).

(This table is available in its entirety in machine-readable form.)

Table 6

UVOT and SEDM Photometry of AT2019wey

\begin{tabular}{lccc}
\hline \hline Date & Instrument & Filter & $m$ \\
\hline 2020-04 Coadd & Swift/UVOT & $B$ & $18.93 \pm 0.17$ \\
2020-04 Coadd & Swift/UVOT & $U$ & $20.16 \pm 0.24$ \\
2020-04 Coadd & Swift/UVOT & $u v m 2$ & $22.55 \pm 0.42$ \\
2020-04 Coadd & Swift/UVOT & $u v w 1$ & $21.17 \pm 0.27$ \\
2020-04 Coadd & Swift/UVOT & $u v w 2$ & $22.86 \pm 0.50$ \\
2020-04 Coadd & Swift/UVOT & $V$ & $18.00 \pm 0.15$ \\
2020-08-05 & Swift/UVOT & $u v m 2$ & $>21.16$ \\
2020-08-09 & Swift/UVOT & $u v m 2$ & $22.16 \pm 0.33$ \\
2020-08-12 & Swift/UVOT & $u v w 2$ & $21.83 \pm 0.21$ \\
2020-08-19 & Swift/UVOT & $U$ & $19.35 \pm 0.06$ \\
2020-08-26 & Swift/UVOT & $u v w 1$ & $20.78 \pm 0.13$ \\
2020-09-02 & Swift/UVOT & $u v m 2$ & $22.12 \pm 0.43$ \\
2020-09-09 & Swift/UVOT & $u v w 2$ & $22.00 \pm 0.24$ \\
2020-09-16 & Swift/UVOT & $U$ & $19.26 \pm 0.07$ \\
2020-09-23 & Swift/UVOT & $u v w 1$ & $20.72 \pm 0.13$ \\
\hline 2020-10-21 & P60/SEDM & $U$ & $19.11 \pm 0.09$ \\
$2020-10-25$ & P60/SEDM & $U$ & $19.21 \pm 0.31$ \\
\hline
\end{tabular}

Note. $m$ is observed magnitude (without extinction correction).

\section{Appendix C Details of Analysis \\ C.1. Extinction Estimation}

The EW of interstellar absorption lines has been observed to be correlated with the amount of reddening. To estimate the extinction of AT2019wey, we produced a summed spectrum from the LRIS and ESI spectra. We did not include DBSP spectra in this analysis

$\overline{17}$ http://www.astro.caltech.edu/ tb/ipac_staff/tab/makee/ 


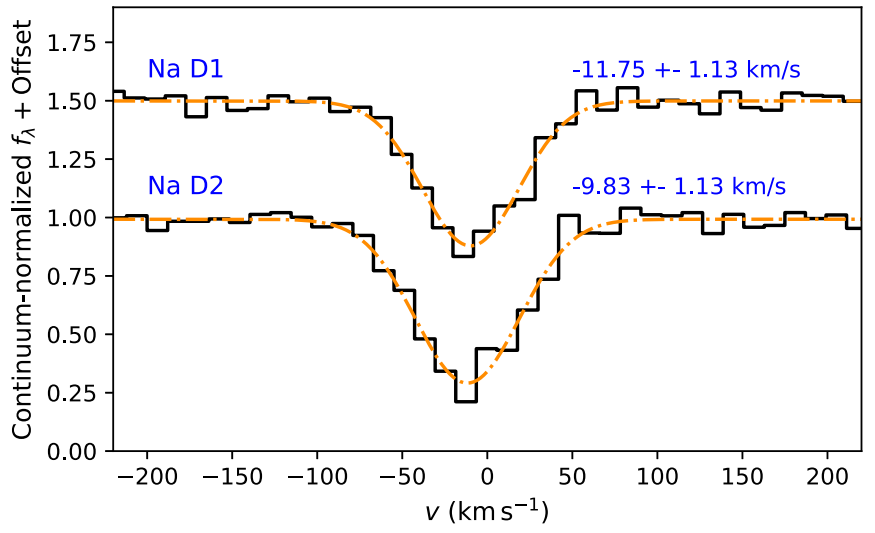

Figure 14. Na I D lines in velocity space fitted with a Gaussian (dashed-dotted lines). The spectrum is heliocentric velocity-corrected.

since the $\mathrm{CCD}$ malfunction resulted in non-astrophysical structures between $5750 \AA$ and $6200 \AA$ in the continuum. This problem prevents EW of spectral lines from being accurately determined from DBSP spectra. The EW of DIB $\lambda \lambda 5780, \lambda 6283$, and Na I D lines were measured from the summed spectrum. As a result, we got $E W(\lambda 5780)=0.56 \pm 0.02 \AA$, and $E W(\lambda 6283)=1.55 \pm$ $0.02 A$. These can be converted to $E(B-V)=0.92 \pm 0.02$ and $1.23 \pm 0.02$ using relations presented by Yuan \& Liu (2012). We got $E W(\mathrm{NaI} \mathrm{D})=1.84 \pm 0.02 \AA$, which can be converted to $E(B-V)=2.01 \pm 0.38$ using the relation in Poznanski et al. (2012)

The inferred $E(B-V)$ values are greater than the total Galactic extinction of $E(B-V)=0.88$ (Schlafly \& Finkbeiner 2011). However, we note that at the measured EW, the calibration uncertainty is large. From Yuan \& Liu (2012, upper panels of Figure 4) and Poznanski et al. (2012, bottom panel of Figure 9), we infer that $E(B-V)$ toward AT2019wey should be $\gtrsim 0.8$.

We also attempt to infer the extinction by assuming that the 6000-10,000 $\AA$ March 23 LRIS spectrum is in the RayleighJeans $(\mathrm{R}-\mathrm{J})$ tail of a blackbody $\left(f_{\lambda} \propto \lambda^{-4}\right.$ when $\left.h \nu \ll k T\right)$, which yields $E(B-V)=1.29$ and a blackbody radius $\left(R_{\mathrm{bb}}\right)$ of

$$
R_{\mathrm{bb}}=\left(4.5 \times 10^{10} \mathrm{~cm}\right)\left(\frac{D}{5 \mathrm{kpc}}\right)\left(\frac{T_{\mathrm{bb}}}{5.0 \times 10^{4} \mathrm{~K}}\right)^{-1 / 2}
$$

Note that this is likely an overestimate of the true extinction (and a lower limit of the outer disk radius), since the optical is only in the R-J limit when $k T \gg 2 \mathrm{eV}\left(T \gg 2 \times 10^{4} \mathrm{~K}\right)$. For instance, for an extinction of $E(B-V) \sim 0.9$, we have

$$
R_{\mathrm{bb}}=\left(1.0 \times 10^{11} \mathrm{~cm}\right)\left(\frac{D}{5 \mathrm{kpc}}\right)\left(\frac{T_{\mathrm{bb}}}{1.1 \times 10^{4} \mathrm{~K}}\right)^{-1 / 2}
$$

\section{C.2. Lower Limit of Distance}

In Appendix C.1, we find that AT2019wey should have an extinction of $0.8 \lesssim E(B-V) \lesssim 1.2$. If this is from diffuse interstellar absorption, the distance of AT2019wey should be greater than $1 \mathrm{kpc}$ using the map of STructuring by Inversion the Local Interstellar Medium (Stilism ${ }^{18}$; Capitanio et al. 2017).

We are able to put a lower limit to the distance using the velocity of the $\mathrm{Na} I \mathrm{D}$ doublets in the ESI spectrum, given that the lines arise from interstellar absorption by a dust cloud along the line-of-sight to AT2019wey. The velocities of D1 and D2

\footnotetext{
${ }^{18}$ https://stilism.obspm.fr/
}

lines were measured to be $-11.75 \pm 1.13 \mathrm{~km} \mathrm{~s}^{-1}$ and $-9.83 \pm$ $1.13 \mathrm{~km} \mathrm{~s}^{-1}$, respectively (see Figure 14). Assuming that the velocity of the dust cloud follows Galactic rotation, we have

$$
V_{\mathrm{obs}, \mathrm{r}}=A d \sin (2 l)
$$

where $A=15.3 \pm 0.4 \mathrm{~km} \mathrm{~s}^{-1} \mathrm{kpc}^{-1}$ is the Oort $A$ constant (Bovy 2017), $l=151.2$ is the Galactic longitude of AT2019wey, and $d$ is distance to the dust cloud. Therefore, Equation (C3) gives $d=0.83 \mathrm{kpc}$.

\section{ORCID iDs}

Yuhan Yao (iD https://orcid.org/0000-0001-6747-8509 S. R. Kulkarni iD https://orcid.org/0000-0001-5390-8563 Kevin B. Burdge (iD https://orcid.org/0000-0002-7226-836X Ilaria Caiazzo (i) https://orcid.org/0000-0002-4770-5388 Kishalay De (iD https://orcid.org/0000-0002-8989-0542 Dillon Dong (i) https://orcid.org/0000-0001-9584-2531 C. Fremling (iD https://orcid.org/0000-0002-4223-103X Mansi M. Kasliwal (ID https://orcid.org/0000-0002-5619-4938 Thomas Kupfer (iD https://orcid.org/0000-0002-6540-1484 Jan van Roestel (iD https://orcid.org/0000-0002-2626-2872 Jesper Sollerman (1D https://orcid.org/0000-0003-1546-6615 Eric C. Bellm (1D https://orcid.org/0000-0001-8018-5348 S. Bradley Cenko (iD https://orcid.org/0000-0003-1673-970X Dmitry A. Duev (D) https://orcid.org/0000-0001-5060-8733 Matthew J. Graham (iD https://orcid.org/0000-0002-3168-0139 Frank J. Masci (i) https://orcid.org/0000-0002-8532-9395 Nicolas Miranda (iD https://orcid.org/0000-0001-6070-7540 Thomas A. Prince (i) https://orcid.org/0000-0002-8850-3627 Reed Riddle (i) https://orcid.org/0000-0002-0387-370X Ben Rusholme (D) https://orcid.org/0000-0001-7648-4142 Maayane T. Soumagnac (iD https://orcid.org/0000-00016753-1488

\section{References}

Armas Padilla, M., Degenaar, N., Russell, D. M., \& Wijnand, s. R. 2013, MNRAS, 428, 3083

Arnaud, K. A. 1996, in ASP Conf. Ser., 101, Astronomical Data Analysis Software and Systems V, ed. V. Systems, G. H. Jacoby, \& J. Barnes (San Francisco, CA: ASP), 17

Astropy Collaboration, Robitaille, T. P., Tollerud, E. J., et al. 2013, A\&A, 558, A33

Bahramian, A., Miller-Jones, J., Strader, J., et al. 2018, Radio/X-ray Correlation Database for X-ray Binaries, Zenodo, doi:10.5281/zenodo. 1252036

Bellm, E. C. 2021, ATel, 14372, 1

Bellm, E. C., Kulkarni, S. R., Graham, M. J., et al. 2019, PASP, 131, 018002

Bellm, E. C., \& Sesar, B. 2016, pyraf-dbsp: Reduction pipeline for the Palomar Double Beam Spectrograph, ASCL, ascl:1602.002

Belloni, T. M., Motta, S. E., \& Muñoz-Darias, T. 2011, BASI, 39, 409

Blagorodnova, N., Neill, J. D., Walters, R., et al. 2018, PASP, 130, 035003

Blandford, R. D., \& Königl, A. 1979, ApJ, 232, 34

Boller, T., Freyberg, M. J., Trümper, J., et al. 2016, A\&A, 588, A103

Bovy, J. 2017, MNRAS, 468, L63

Callanan, P. J., Garcia, M. R., McClintock, J. E., et al. 1995, ApJ, 441, 786

Cao, H., Frey, S., Gabanyi, K., et al. 2020a, ATel, 13984, 1

Cao, H., Giroletti, M., Migliori, G., \& Frey, S. 2020b, ATel, 14168, 1

Capitanio, L., Lallement, R., Vergely, J. L., Elyajouri, M., \& Monreal-Ibero, A. 2017, A\&A, 606, A65

Cardelli, J. A., Clayton, G. C., \& Mathis, J. S. 1989, ApJ, 345, 245

Cenko, S. B., Fox, D. B., Moon, D.-S., et al. 2006, PASP, 118, 1396

Chiang, C. Y., Done, C., Still, M., \& Godet, O. 2010, MNRAS, 403, 1102

Condon, J. J., Cotton, W. D., Greisen, E. W., et al. 1998, AJ, 115, 1693

Corbel, S., Nowak, M. A., Fender, R. P., Tzioumis, A. K., \& Markoff, S. 2003, A\&A, 400, 1007

Corral-Santana, J. M., Casares, J., Muñoz-Darias, T., et al. 2013, Sci, 339,1048 
Cushing, M. C., Vacca, W. D., \& Rayner, J. T. 2004, PASP, 116, 362

Done, C., Gierliński, M., \& Kubota, A. 2007, A\&ARv, 15, 1

Dubus, G., Kim, R. S. J., Menou, K., Szkody, P., \& Bowen, D. V. 2001, ApJ, 553, 307

Dubus, G., Lasota, J.-P., Hameury, J.-M., \& Charles, P. 1999, MNRAS, 303, 139

Esin, A. A., Lasota, J. P., \& Hynes, R. I. 2000, A\&A, 354, 987

Fender, R. P. 2001, MNRAS, 322, 31

Fender, R. P., Belloni, T. M., \& Gallo, E. 2004, MNRAS, 355, 1105

Flewelling, H. A., Magnier, E. A., Chambers, K. C., et al. 2020, ApJS, 251, 7

Foreman-Mackey, D., Hogg, D. W., Lang, D., \& Goodman, J. 2013, PASP, 125,306

Fremling, C., Sollerman, J., Taddia, F., et al. 2016, A\&A, 593, A68

Froning, C. S., Maccarone, T. J., France, K., et al. 2014, ApJ, 780, 48

Gehrels, N., Chincarini, G., Giommi, P., et al. 2004, ApJ, 611, 1005

Gierliński, M., Done, C., \& Page, K. 2008, MNRAS, 388, 753

Gierliński, M., Done, C., \& Page, K. 2009, MNRAS, 392, 1106

Graham, M. J., Kulkarni, S. R., Bellm, E. C., et al. 2019, PASP, 131, 078001

Harding, L. K., Hallinan, G., Milburn, J., et al. 2016, MNRAS, 457, 3036

Heasarc 2014, HEAsoft: Unified Release of FTOOLS and XANADU, ascl: 1408.004

Horne, K., \& Marsh, T. R. 1986, MNRAS, 218, 761

Hunter, J. D. 2007, CSE, 9, 90

Jiménez-Ibarra, F., Muñoz-Darias, T., Casares, J., Armas Padilla, M., \& Corral-Santana, J. M. 2019, MNRAS, 489, 3420

Kasliwal, M. M., Cannella, C., Bagdasaryan, A., et al. 2019, PASP, 131, 038003

Kaur, R., Kaper, L., Ellerbroek, L. E., et al. 2012, ApJL, 746, L23

Kawamuro, T., Negoro, H., Yoneyama, T., et al. 2018, ATel, 11399, 1

Kupfer, T., Prince, T. A., van Roestel, J., et al. 2021, MNRAS, arXiv:2105.02758

La Dous, C. 1989, A\&A, 211, 131

Lacy, M., Baum, S. A., Chandler, C. J., et al. 2020, PASP, 132, 035001

Lasota, J. P., Dubus, G., \& Kruk, K. 2008, A\&A, 486, 523

Law, N. M., Kulkarni, S. R., Dekany, R. G., et al. 2009, PASP, 121, 1395

Masci, F. J., Laher, R. R., Rusholme, B., et al. 2019, PASP, 131, 018003

Mata Sánchez, D., Muñoz-Darias, T., Casares, J., Corral-Santana, J. M., \& Shahbaz, T. 2015, MNRAS, 454, 2199

Matsuoka, M., Kawasaki, K., Ueno, S., et al. 2009, PASJ, 61, 999

McClintock, J. E., \& Remillard, R. A. 2006, in Black Hole Binaries, ed. W. Lewin \& M. van der Klis, Vol. 39 (Cambridge: Cambridge Univ. Press), 157

McKinney, W. 2010, in Proc. 9th Python in Science Conf., Austin, TX, 18-23 August 2010, ed. S. van der Walt \& J. Millman, 51

McMullin, J. P., Waters, B., Schiebel, D., Young, W., \& Golap, K. 2007, in ASP Conf. Ser., 376, Astronomical Data Analysis Software and Systems XVI, ed. R. A. Shaw, F. Hill, \& D. J. Bell (San Francisco, CA: ASP), 127

Mereminskiy, I., Medvedev, P., Semena, A., et al. 2020, ATel, 13571, 1

Meyer-Hofmeister, E. 2004, A\&A, 423, 321

Minkowski, R. L., \& Abell, G. O. 1963, in The National Geographic SocietyPalomar Observatory Sky Survey, ed. K. A. Strand (Chicago: Univ. Chicago Press), 481

Mitsuda, K., Inoue, H., Koyama, K., et al. 1984, PASJ, 36, 741

Morales-Rueda, L., \& Marsh, T. R. 2002, MNRAS, 332, 814

Narayan, R., \& Yi, I. 1994, ApJL, 428, L13

Narayan, R., \& Yi, I. 1995, ApJ, 452, 710

Negoro, H., Kohama, M., Serino, M., et al. 2016, PASJ, 68, S1

Neustroev, V. V., Veledina, A., Poutanen, J., et al. 2014, MNRAS, 445, 2424

Oke, J. B., Cohen, J. G., Carr, M., et al. 1995, PASP, 107, 375

Oke, J. B., \& Gunn, J. E. 1982, PASP, 94, 586

Osaki, Y. 1996, PASP, 108, 39

Patterson, J., \& Raymond, J. C. 1985, ApJ, 292, 550
Pavlinsky, M., Tkachenko, A., Levin, V., et al. 2021, A\&A, 650, A42 Perley, D. A. 2019, PASP, 131, 084503

Perley, R. A., Chandler, C. J., Butler, B. J., \& Wrobel, J. M. 2011, ApJL, 739, L1

Poutanen, J., \& Veledina, A. 2014, SSRv, 183, 61

Poznanski, D., Prochaska, J. X., \& Bloom, J. S. 2012, MNRAS, 426, 1465

Predehl, P., Andritschke, R., Arefiev, V., et al. 2021, A\&A, 647, A1

Rahoui, F., Tomsick, J. A., Coriat, M., et al. 2015, ApJ, 810, 161

Rau, A., Kulkarni, S. R., Law, N. M., et al. 2009, PASP, 121, 1334

Reid, I. N., Brewer, C., Brucato, R. J., et al. 1991, PASP, 103, 661

Remillard, R. A., \& McClintock, J. E. 2006, ARA\&A, 44, 49

Rigault, M., Neill, J. D., Blagorodnova, N., et al. 2019, A\&A, 627, A115

Roming, P. W. A., Kennedy, T. E., Mason, K. O., et al. 2005, SSRv, 120, 95

Russell, D. M., Fender, R. P., Hynes, R. I., et al. 2006, MNRAS, 371, 1334

Russell, T. D., Soria, R., Motch, C., et al. 2014, MNRAS, 439, 1381

Schlafly, E. F., \& Finkbeiner, D. P. 2011, ApJ, 737, 103

Schwarzenberg-Czerny, A. 1998, BaltA, 7, 43

Shahbaz, T., \& Kuulkers, E. 1998, MNRAS, 295, L1

Shakura, N. I., \& Sunyaev, R. A. 1973, A\&A, 500, 33

Shaw, A. W., Charles, P. A., Bird, A. J., et al. 2013, MNRAS, 433, 740

Shaw, A. W., Charles, P. A., Casares, J., \& Hernández Santisteban, J. V. 2016, MNRAS, 463, 1314

Shaw, A. W., Tetarenko, B. E., Dubus, G., et al. 2019, MNRAS, 482, 1840

Sheinis, A. I., Bolte, M., Epps, H. W., et al. 2002, PASP, 114, 851

Smith, K. W., Smartt, S. J., Young, D. R., et al. 2020, PASP, 132, 085002

Soria, R., Wu, K., \& Hunstead, R. W. 2000, ApJ, 539, 445

Sunyaev, R., Arefiev, V., Babyshkin, V., et al. 2021, arXiv:2104.13267

Tetarenko, B. E., Dubus, G., Marcel, G., Done, C., \& Clavel, M. 2020, MNRAS, 495, 3666

Tetarenko, B. E., Sivakoff, G. R., Heinke, C. O., \& Gladstone, J. C. 2016 , ApJS, 222, 15

Tonry, J., Denneau, L., Heinze, A., et al. 2019, TNSTR, 2019-2553, 1

Torres, M. A. P., Callanan, P. J., Garcia, M. R., et al. 2002, ApJ, 569, 423

Torres, M. A. P., Steeghs, D., Jonker, P. G., Thompson, I., \& Soderberg, A. M. 2009, ATel, 2268, 1

Truemper, J. 1982, AdSpR, 2, 241

Tuchman, Y., Mineshige, S., \& Wheeler, J. C. 1990, ApJ, 359, 164

Tucker, M. A., Shappee, B. J., Holoien, T. W. S., et al. 2018, ApJL, 867, L9

Vacca, W. D., Cushing, M. C., \& Rayner, J. T. 2003, PASP, 115, 389

van Paradijs, J., \& McClintock, J. E. 1994, A\&A, 290, 133

VanderPlas, J. T. 2018, ApJS, 236, 16

Virtanen, P., Gommers, R., Oliphant, T. E., et al. 2020, Nature Methods, 17,261

Voges, W., Aschenbach, B., Boller, T., et al. 1999, A\&A, 349, 389

Warner, B. 1995, Cataclysmic Variable Stars, Cambridge Astrophysics (Cambridge: Cambridge Univ. Press)

Waters, C. Z., Magnier, E. A., Price, P. A., et al. 2020, ApJS, 251, 4

Wu, Y. X., Yu, W., Li, T. P., Maccarone, T. J., \& Li, X. D. 2010, ApJ, 718,620

Yadlapalli, N., Ravi, V., Yao, Y., Kulkarni, S. R., \& Brisken, W. 2021, ApJL, 909, L27

Yao, Y., De, K., Kasliwal, M. M., et al. 2020b, ApJ, 900, 46

Yao, Y., Kulkarni, S. R., Gendreau, K. C., et al. 2020a, ApJ, 920, 121

Yuan, F., Cui, W., \& Narayan, R. 2005, ApJ, 620, 905

Yuan, F., \& Narayan, R. 2014, ARA\&A, 52, 529

Yuan, H. B., \& Liu, X. W. 2012, MNRAS, 425, 1763

Zhang, G. B., Bernardini, F., Russell, D. M., et al. 2019, ApJ, 876, 5

Zhang, S.-N. 2013, FrPhy, 8, 630

Zurita, C., Durant, M., Torres, M. A. P., et al. 2008, ApJ, 681, 1458

Zurita, C., Sánchez-Fernández, C., Casares, J., et al. 2002, MNRAS, 334, 999 